\title{
Infrared observations of NGC 3603
}

\section{A $11.9 \mu \mathrm{m}$ and $18 \mu \mathrm{m}$ survey ${ }^{\star}$}

\author{
D. E. A. Nürnberger ${ }^{1,2}$ and T. Stanke ${ }^{3}$ \\ 1 European Southern Observatory, Casilla 19001, Santiago 19, Chile \\ 2 Institut für Theoretische Physik und Astrophysik, Univ. Würzburg, Am Hubland, 97074 Würzburg, Germany \\ 3 Max-Planck-Institut für Radioastronomie, Auf dem Hügel 69, 53121 Bonn, Germany
}

Received 2 July 2002 / Accepted 23 December 2002

\begin{abstract}
We present results of the first sub-arcsec resolution mid infrared survey of the southern hemisphere giant $\mathrm{H}_{\text {II }}$ region NGC 3603. We have observed selected fields in the vicinity of the OB cluster at wavelengths of $11.9 \mu \mathrm{m}$ and $18 \mu \mathrm{m}$ using TIMMI 2 mounted on the ESO $3.6 \mathrm{~m}$ telescope. These fields comprise areas with dense molecular cores, embedded near infrared sources as well as several $\mathrm{OH}, \mathrm{H}_{2} \mathrm{O}$ and $\mathrm{CH}_{3} \mathrm{OH}$ maser sources, which give indications of ongoing star formation processes. We report the detection of 36 mid infrared point sources and additionally provide flux measurements for 42 knots of diffuse emission.

In the area surveyed the protostar IRS 9A is found to be the most luminous source at both $11.9 \mu \mathrm{m}$ and $18 \mu \mathrm{m}$. Located in its immediate vicinity two more sources (IRS 9B and IRS 9C) also exhibit significant $11.9 \mu \mathrm{m}$ and $18 \mu \mathrm{m}$ emission, thus providing further indications for IRS 9 being an association of protostars in its own right. Several other $11.9 \mu \mathrm{m}$ point sources are related to near infrared sources with strong $K$-band excess emission and/or to maser sources, which classifies them as young sources, too. In contrast, the second strongest $11.9 \mu \mathrm{m}$ source, IRS 4, appears to be in a more evolved stage.

Towards the center of the OB cluster we observe mid infrared emission arising from the three Wolf-Rayet stars WR 43abc, providing evidence for dust production and/or the presence of plasma in their circumstellar envelopes. Spread all over the cluster, we detect a number of sources with mid infrared fluxes close to the sensitivity limit ( 0.01 Jy) of our $11.9 \mu \mathrm{m}$ data, which apparently have very red $K-N$ colours. We suggest that these sources are circumstellar disks which are externally heated by the nearby massive stars.

Towards the south and west of the OB cluster, large amounts of diffuse emission are found closely correlated with ionized material. We identify at least 7 shocks and ionization fronts, reflecting the enormous impact of the fast stellar winds and ionizing photons, originating from the massive cluster stars, on the adjacent gas and dust. This is impressively emphasized by the shocked and ionized material associated with the heads of the two prominent pillars. Both pillars are easily seen in our $11.9 \mu \mathrm{m}$ and $18 \mu \mathrm{m}$ data: the western one rather prominent in emission, the eastern one more pronounced in absorption against a strong diffuse mid infrared background.

Among those sources, for which our data do not reveal any point-like mid infrared counterpart, are IRS 1 as well as the three "proplyds". However, at least for "proplyd" 3 we detect extended, rim-like $11.9 \mu \mathrm{m}$ emission. Therefore, we consider it likely that NGC 3603's "proplyds" simply represent scaled-down versions of the neighbouring pillars, i.e. remnant density enhancements of the pristine molecular cloud which to date were able to resist the ionizing and photoevaporating radiation from the nearby OB stars.
\end{abstract}

Key words. surveys - stars: early-type - stars: formation - stars: pre-main sequence - ISM: H II regions ISM: individual objects: NGC 3603

\section{Introduction}

The NGC 3603 star cluster is located beyond the tangential point of the Carina arm, at a distance of about $7 \pm 1 \mathrm{kpc}$ (Goss et al. 1972; Moffat 1974, 1983; Van den Bergh 1978;

Send offprint requests to: D. E. A. Nürnberger,

e-mail: dnuernbe@eso.org

* Based on data sets obtained at the European Southern Observatory on La Silla, Chile.
Clayton 1986; Melnick et al. 1989; Crowther \& Dessart 1998; De Pree et al. 1999; Pandey et al. 2000). Apart from the Arches cluster (Figer et al. 1999) near the Galactic Center, it shows the highest density of high mass stars known in the Galaxy (Melnick et al. 1989; Moffat et al. 1994; Drissen et al. 1995; Hofmann et al. 1995). On its own, the Trapezium-like system HD 97950 in the cluster core contains 3 luminous hydrogenrich WNL stars, 6 O3 stars and many other late O type stars at an age of about 2...3 Myr (Melnick et al. 1989; 




Fig. 1. Central 13'. $5 \times 13^{\prime} .5$ of our Wide Field Imager (WFI) data. The three-colour image combines observations taken in H $\alpha$ (red), [S II] (green) and $8160 \AA$ continuum (blue) filters. The areas surveyed with the TIMMI 2 camera at $11.9 \mu \mathrm{m}$ and $18 \mu \mathrm{m}$ are outlined by straight and dashed lines, respectively.

Hofmann et al. 1995). The combined effort of these hot stars has a significant influence on the surrounding gas and dust: on the one hand by providing a huge amount of ionizing photons (Lyman continuum flux $\sim 10^{51} \mathrm{~s}^{-1}$; Kennicutt 1984; Drissen et al. 1995) and on the other hand by compressing the adjacent molecular cloud (Nürnberger 2002; Nürnberger et al. 2002b) through fast stellar winds (up to several $100 \mathrm{~km} \mathrm{~s}^{-1}$; Balick et al. 1980).

This is impressively demonstrated by Fig. 1 which shows the central part $\left(13^{\prime} .5 \times 13^{\prime} .5\right)$ of the NGC 3603 H II region. The three-colour image combines optical $\mathrm{H} \alpha$ (red), [S II] (green) and $8160 \AA$ continuum (blue) data which were obtained with the Wide Field Imager (WFI) at the ESO/MPG 2.2-m telescope on La Silla. The entire field-of-view is dominated by diffuse emission from ionized material, partly exhibiting sharp contrasts to opaque patches of cold gas and dust in the foreground. Close to the central cluster, the synergy of both energetic Lyman continuum photons and stellar winds evaporates and disperses density enhancements of the interstellar medium, giving rise to pillar-like and cometary shaped phenomena (Brandner et al. 2000). Furthermore, stellar winds are considered the driving sources of a gas-free "bubble" which 
expands with velocities of about $50 \mathrm{~km} \mathrm{~s}^{-1}$ into the ambient medium (Balick et al. 1980; Clayton 1986, 1990).

Observations at mid infrared (IR) wavelengths are tracing properties of highly ionized gas and/or warm dust - typically, the temperatures are a few $100 \mathrm{~K}$ - and hence provide an important tool to probe the impact of massive stars on their interstellar medium. About 25 years ago, Frogel et al. (1977) have presented first broad band mid IR imaging data of NGC 3603, although very limited both in angular resolution and in sensitivity. Their study was followed 5 years later by the work of Lacy et al. (1982) who used mid IR emission lines to investigate the structure of and the excitation conditions within the NGC 3603 H II region. Ever since, great improvements were achieved in mid IR instrumentation as well as in mid IR observing techniques. Thus, considering the often quoted importance of NGC 3603 for the understanding of extragalactic starburst regions, it is surprising that during all these years no attempt was made to gain more detailed insight at mid IR wavelengths. Very recently, we have undertaken a survey of NGC 3603 at $11.9 \mu \mathrm{m}$ and $18 \mu \mathrm{m}$ using the TIMMI 2 camera. We present the results of this imaging survey in this paper.

\section{Survey fields}

The fields of our TIMMI 2 survey of NGC 3603 are indicated in Fig. 1: straight and dashed lines outline the areas observed in the N11.9 and Q1 filter, respectively.

The fields $11.9-1$ and 18-1 primarily cover intensely ionized gas and dust in the immediate vicinity of the OB cluster, including the two prominent pillars and the proplyd-like sources (Brandner et al. 2000) as well as the IRS 9 association of candidate-protostars (Nürnberger et al. 2002a). In addition, field 11.9-1 also traces the southern parts of the molecular cloud (Nürnberger et al. 2002b) up to a distance of about $5^{\prime} .5$ from the center of the OB cluster.

The fields 11.9-2 and 18-2 are centered close to a number of maser sources $\left(\mathrm{OH}, \mathrm{H}_{2} \mathrm{O}\right.$ and $\mathrm{CH}_{3} \mathrm{OH}$; Caswell \& Haynes 1987; Caswell 1998; Caswell et al. 1989, 1995) which are located about $5^{\prime} .5$ towards the north of HD 97950 on the edge of a massive molecular clump (Nürnberger et al. 2002b). The appearance of maser sources is usually thought to be indicative for ongoing star formation processes.

\section{Observations and data reduction}

A first set of NGC 3603 frames using the N11.9 filter $\left(\lambda_{\mathrm{c}}=11.59 \mu \mathrm{m}, \Delta \lambda_{50 \%}= \pm 0.60 \mu \mathrm{m}\right)$ was obtained on January 22nd-24th 2001 with the Thermal Infrared Multi-Mode Instrument 2 (TIMMI2; Reimann et al. 2000) mounted on ESO's $3.6 \mathrm{~m}$ telescope atop La Silla, Chile. However, these data were amongst the very first TIMMI 2 science data and suffered from a high noise level, most likely due to a dirty dichroic and a still embryonic setup of the overall camera system. After successful cleaning of TIMMI 2's optical components during September 2001, a second set of $11.9 \mu \mathrm{m}$ data was observed on December 28th-31st 2001 with the thermal background at much lower levels than in the earlier data. In addition, we performed imaging with the Q1 filter $\left(\lambda_{\mathrm{c}}=17.75 \mu \mathrm{m}, \Delta \lambda_{50 \%}= \pm 0.40 \mu \mathrm{m}\right)$. During all five nights the sky was clear with a typical optical seeing of about 0 ". 5 , resulting in diffraction limited images at $11.9 \mu \mathrm{m}$ and $18 \mu \mathrm{m}$, and a good to medium IR transparency (water vapour between $12 \%$ and $75 \%$ ).

The TIMMI 2 camera features a Raytheon $320 \times 240$ pixel Si:As array, providing a field-of-view of $96^{\prime \prime} \times 72^{\prime \prime}$ (at 0 ".296 per pixel) for our N11.9 data and $64^{\prime \prime} \times 48^{\prime \prime}$ (at $0^{\prime \prime} .202$ per pixel) for our Q1 data, respectively. Chopping throws (in $\mathrm{N}-\mathrm{S}$ direction) and nodding throws (in $\mathrm{E}-\mathrm{W}$ direction during the first run, in $\mathrm{N}-\mathrm{S}$ direction during the second run) were set to $10^{\prime \prime}$ for the calibration frames and varied between $10^{\prime \prime}$ and $20^{\prime \prime}$ for the science frames. The total exposure time per frame (i.e. including all chopping and all nodding positions) was 1.8-6.7 min for field N11.9-1, 2.2-8.1 min for field N11.92, 6.5 min for field Q18-1 and about 9.7 min for field Q18-2.

The data reduction - which we performed within IRAF, IDL and MIDAS - is not a trivial issue at mid IR wavelengths, in particular for extended structures. To get rid of the high thermal background noise, mid IR observations require a chopping and nodding technique which produces not only a positive signal at the location (ON position) of a given astronomical source, but also negative features if the chopper throw is chosen such that the source remains within the field-of-view in the OFF positions. While it is fairly easy to identify the positive and negative signals for point sources, this task is very difficult for areas containing highly extensive and structured emission as it turned out to be the case in NGC 3603. In order to preserve the information on both the point sources and on the complex spatial distribution of diffuse emission, our data were processed in two ways.

On the one hand, a "nulling" technique was used which properly shifts and adds / subtracts the frames of corresponding chopping and nodding positions according to their relative offsets. The aim of this (iterative) process is to get rid of all the negative signals by gradually moving them out of the scientifically relevant field-of-view. Any remaining non-negligible residuals (primarily caused by imperfect point spread functions as well as insufficient frame alignments) are then compensated by applying minmax or sigma clipping criteria while combining all resulting frames.

On the other hand the iterative-inversion image restoration method outlined by Bertero et al. (2000) was adapted to our problem. First, the algorithm was extended to also allow for nodding perpendicular to the chopping direction in order to restore the data taken in January 2001. Second, instead of applying the algorithm on each individual frame and combining the results into a final mosaic after many iterations, only one iteration per frame was done before an intermediate mosaic was created. Then, the input model for the next iteration was extracted from this mosaic, thus using for each step the full available information. In order not to degrade the much higher quality data taken in December 2001, while still making use of the poorer January 2001 data as good as possible, the frames were combined into mosaics weighting them as $r m s^{-2}$, with the $r m s$ determined from the original unrestored data. 
Table 1. ISO standard stars used for the photometric calibration, with N11.9 and Q1 fluxes taken from SED models by Cohen (1998).

\begin{tabular}{llccccc}
\hline \hline Calibrator & Alias & SpT & $\begin{array}{c}V \\
{[\mathrm{mag}]}\end{array}$ & $\begin{array}{c}F_{12 \mu \mathrm{m}}^{\mathrm{IRAS}} \\
{[\mathrm{Jy}]}\end{array}$ & $\begin{array}{c}F_{\mathrm{N} 11.9} \\
{[\mathrm{Jy}]}\end{array}$ & $\begin{array}{c}F_{\mathrm{Q} 1} \\
{[\mathrm{Jy}]}\end{array}$ \\
\hline HD 4128 & & K0III & $2.04 \mathrm{v} ?$ & 39.31 & 39.4 & 11.6 \\
HD 29139 & $\alpha$ Tau & K5III & $0.85 \mathrm{v}$ & 699.7 & 491 & 14 \\
HD 32887 & $\epsilon$ Lep & K4III & $3.2 \mathrm{v}$ & 56.8 & 41.5 & 12.3 \\
HD 47105 & & A0IV & 1.90 & 5.04 & 5.23 & 1.55 \\
HD 81420 & & K5III & 5.60 & 7.41 & 5.15 & 1.53 \\
HD 81797 & $\alpha$ Hya & K3III & $2.00 \mathrm{v}$ & 157.6 & 101 & 29.5 \\
\hline
\end{tabular}

The image restoration method by Bertero et al. works best if the chopping and nodding direction is exactly along the columns (or rows) of the array, and if the chopping/nodding amplitude corresponds to an integer number of pixels. As this was not the case with our original data, they had to be rotated (typically by about $0.2-0.3^{\circ}$ ) and rebinned. After each iteration the resulting frames were rotated and rebinned back to their original orientation and pixel sizes, and then combined into a temporary mosaic. To get the input model frame for the next iteration, the proper part of the intermediate mosaic was taken and rotated and rebinned the same way as the original input frame. This procedure significantly reduces artefacts resulting from the imperfect alignment of the chopping/ nodding direction along array columns/rows as well as from chopping/nodding throws with amplitudes being non-integer multiples of pixel units.

However, due to the repeated rotations and rebinnings, the point-spread function was seen to degrade considerably. To keep this effect as small as possible, the relaxation parameter $\tau$ used in the restoration algorithm was increased to 0.15 in order to decrease the number of iterations needed to obtain a given result. In addition, negative and positive residuals (of strong point sources and strong diffuse emission, in particular) remain in the final mosaic, disturbing the identification and photometry of real sources.

\section{Photometric and astrometric calibration}

Photometry of our detected sources was performed on the December 2001 data set, with the sole exception of NS 4 which was only covered by the January 2001 data. For photometric calibration the ISO standard stars HD 29139, HD 47105 and HD 4128, HD 32887, HD 81420, HD 81797 were used during the first and second observing run, respectively. Some of their parameters are summarized in Table 1. Estimates of their $F_{\mathrm{N} 11.9}$ and $F_{\mathrm{Q} 1}$ fluxes are taken from SED models by Cohen (1998), with typical flux errors of about $3 \%(1 \sigma)$. Each calibrator was observed twice during each night, showing flux variations in the range of the theoretical flux errors, i.e. about $3 \%$. The nightto-night flux variations were measured to be larger, namely of the order $10 \%$ at $11.9 \mu \mathrm{m}$ and up to $20 \%$ at $18 \mu \mathrm{m}$.

Astrometric calibration for point sources and knot-like features identified in the fields $11.9-1$ and 18-1 was performed relative to IRS 9A $\left(\mathrm{RA}_{\mathrm{J} 2000}=11^{\mathrm{h}} 15^{\mathrm{m}} 11.34, \mathrm{DEC}_{\mathrm{J} 2000}=\right.$ $-61^{\circ} 16^{\prime} 45^{\prime \prime}$. 2 ; ICRS frame). Its accuracy is limited by the pixel scale of the TIMMI 2 detector and hence supposed to be 0.04 in right ascension and $0^{\prime \prime} .3$ in declination. An independent position check was achieved by registering IRS 9A on a deep VLT + ISAAC $K_{\mathrm{s}}$ band mosaic (Nürnberger \& Petr 2002).

In order to determine the positions of the sources in the northern fields 11.9-2 and 18-2 the following procedure was applied. First we determined pointing offsets of the telescope by observing IRS 9A. The telescope was then pointed on the coordinates of NS 2 as derived from observations during the first night, and by additionally applying the pointing offsets NS 2 was brought to within a few pixels of the central pixel - which gives the reference position - of the array. Thus, we estimate that the positions for the northern fields are accurate to a few pixels or $1-2^{\prime \prime}$.

\section{Imaging results}

In Fig. 2 we present the imaging result for field 11.9-1, with point sources and knot-like features annotated (see the corresponding entries in Table 2) and with filaments properly outlined. The position of the NGC 3603 OB cluster is indicated by an open circle and asterisks mark positions of prominent IR sources as given by Frogel et al. (1977). OH maser (Braz \& Epchtein 1983; Caswell \& Haynes 1987; Caswell 1998) and $\mathrm{H}_{2} \mathrm{O}$ maser (Caswell et al. 1989) are indicated by squares and triangles, respectively. Also note the three crosses which give the positions of cometary shaped objects, identified - and labeled "proplyds" - by Brandner et al. (2000).

Additionally, we used the image restoration method of Bertero et al. (2000) on the same data set to produce Fig. 3. A detailed comparison between the two plots of field 11.9-1 illustrates the (dis)advantages of the two data reduction methods. The "nulling" technique allows a good truncation of artefacts and residuals, in particular important in areas of widely extended diffuse emission, but the detection of very faint sources is rather difficult. In contrast, the restoration method of Bertero et al. (2000) provides a powerful tool for the reliable detection of point sources at fairly low signal-to-noise levels but suffers from the incomplete removal of residuals (in particular critical in cases where strong extended emission is present), thus limiting the accuracy of the photometry. Hence, both methods nicely complement each other.

In Fig. 4 we zoom-in on the area located immediately towards the south of the OB cluster (as outlined by the dashed box in Fig. 2 and basically comprising the field 18-1) and compare the TIMMI 2 data with those from optical and near IR imaging. While panel a shows combined 


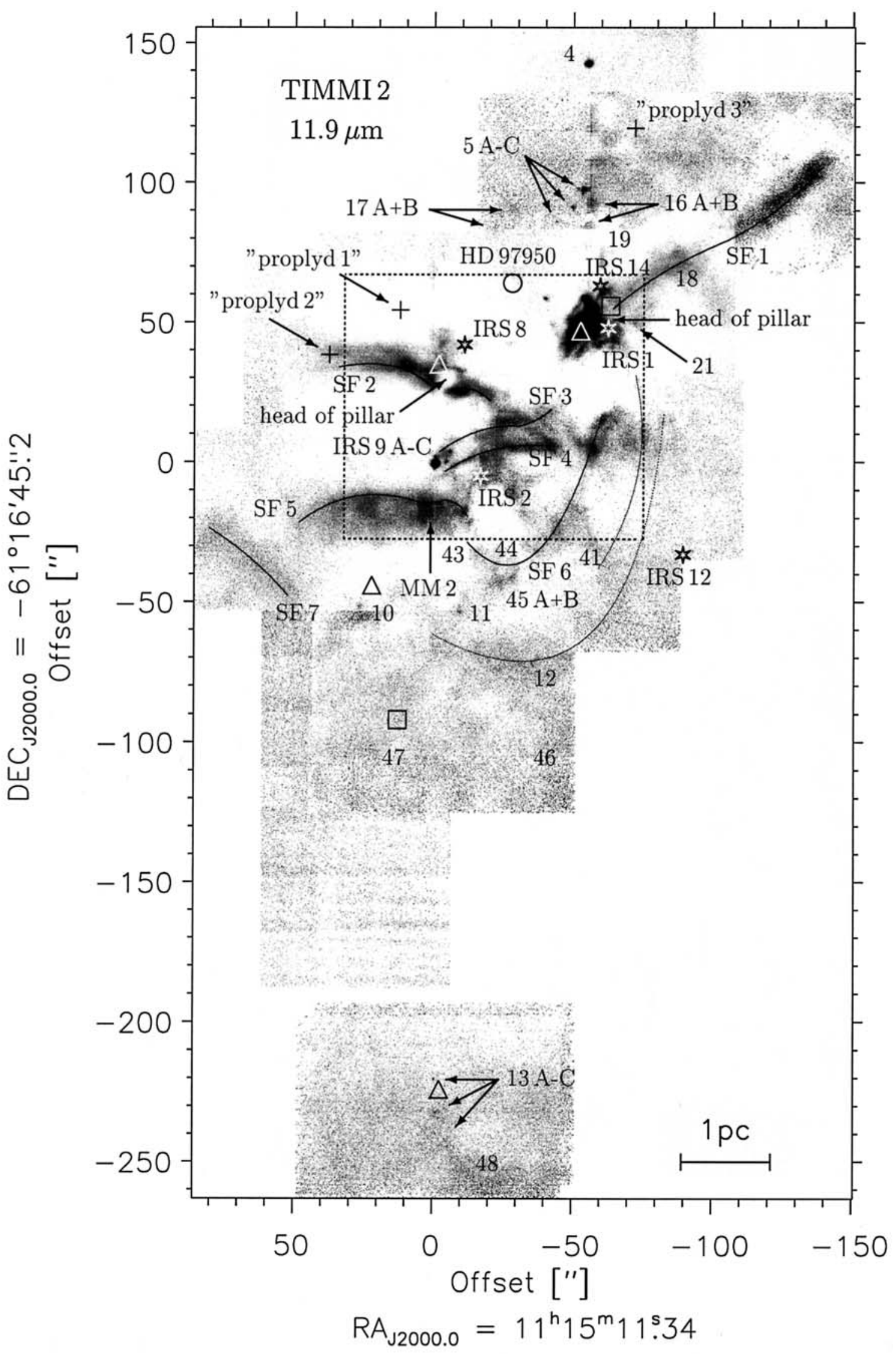

Fig. 2. Imaging result obtained with TIMMI 2 at $11.9 \mu \mathrm{m}$ for the field 11.9-1, using the "nulling" (shift and add/ subtract) technique described in Sect. 3. (Un)detected point sources and prominent features of diffuse emission are annotated. Numbers refer to individual entries of Table 2. For the meaning of the other symbols please see the text. The dashed box indicates the field-of-view which is shown in more detail in Fig. 4c. 


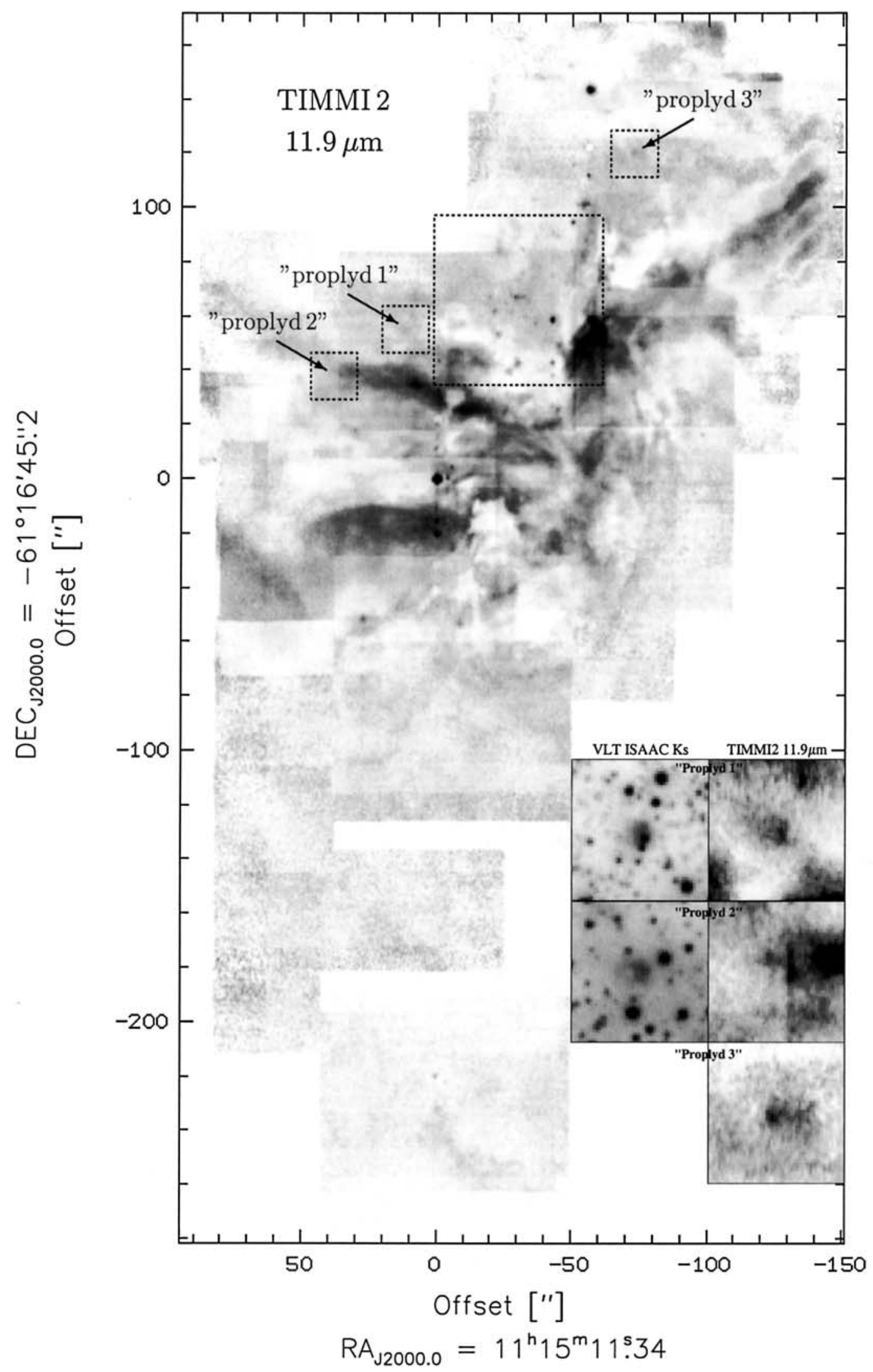

Fig. 3. Same data set as shown in Fig. 2 but this time reduced with the iterative-inversion image restoration method described in the text (Sect. 3). Careful comparison to the result obtained by using the "nulling" technique reveals the (dis)advantages of each technique in terms of residuals, artefacts and faint sources. The large dashed box outlines the central part of the OB cluster displayed in Fig. 6, while the small dashed boxes (centered on the "proplyd" positions) indicate those areas covered by the inserts. 

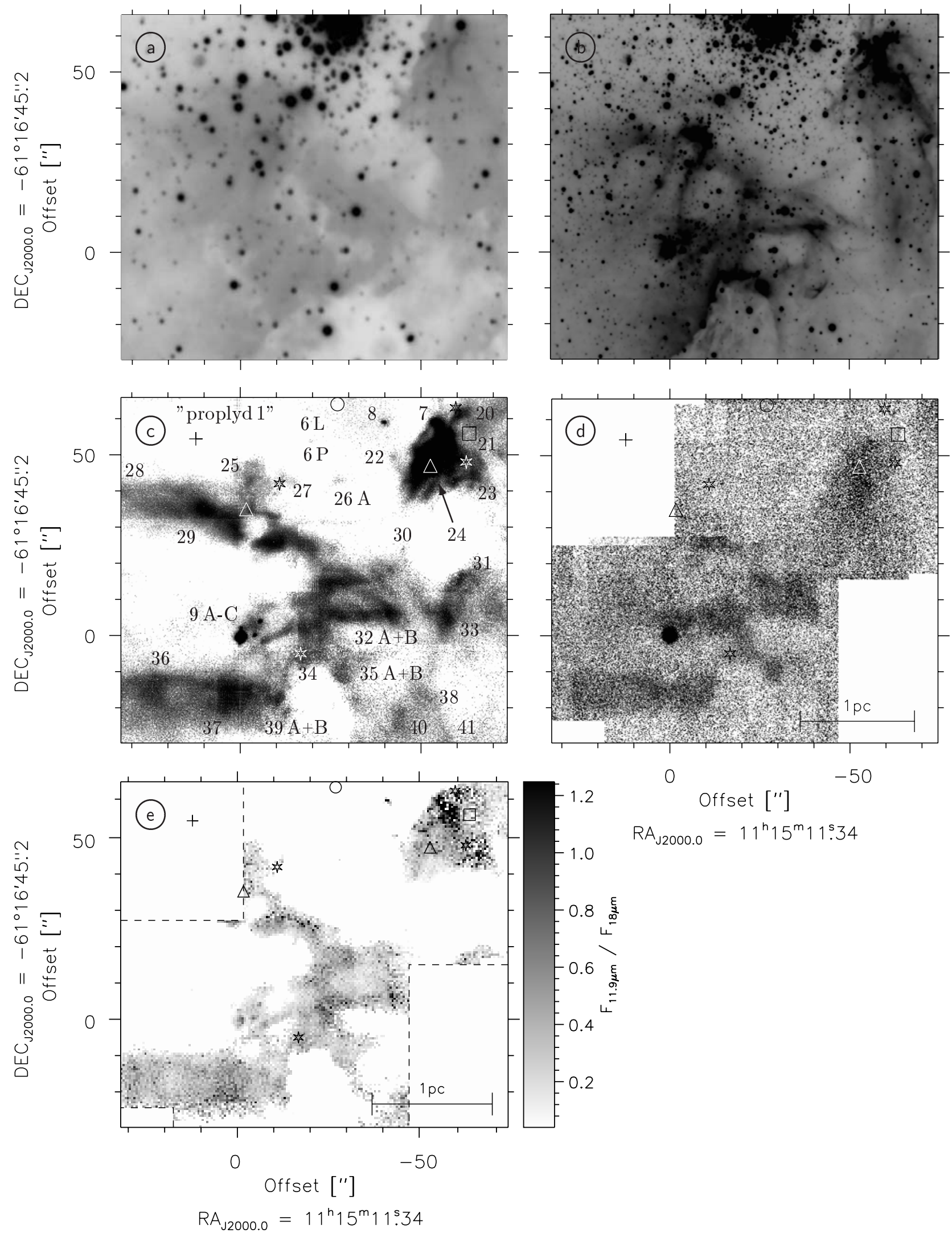

0

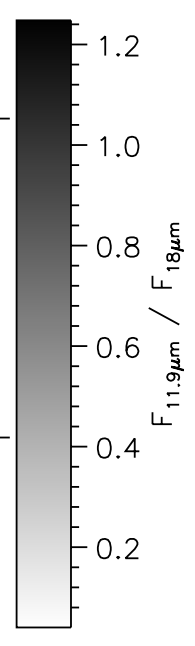

$$
\begin{gathered}
\text { Offset ["] } \\
\mathrm{RA}_{\mathrm{J} 2000.0}=11^{\mathrm{h}} 15^{\mathrm{m}} 11.34
\end{gathered}
$$

$-50$

Fig. 4. Zoom-in on the area towards the south of the NGC 3603 OB cluster. Panels a) and b) show optical (H $\alpha+[\mathrm{S} \mathrm{II}]+8160 \AA$ cont) and $\mathrm{K}_{\mathrm{s}}$ data obtained with WFI at the ESO/MPG 2.2-m telescope (Fig. 1) and ISAAC at the VLT Antu (see Nürnberger \& Petr-Gotzens 2002), respectively. The corresponding $11.9 \mu \mathrm{m}$ and $18 \mu \mathrm{m}$ TIMMI 2 images are presented in panels c) and d). Panel e) gives the $11.9 \mu \mathrm{m} / 18 \mu \mathrm{m}$ flux ratio which is an indicator for the dust temperature. 

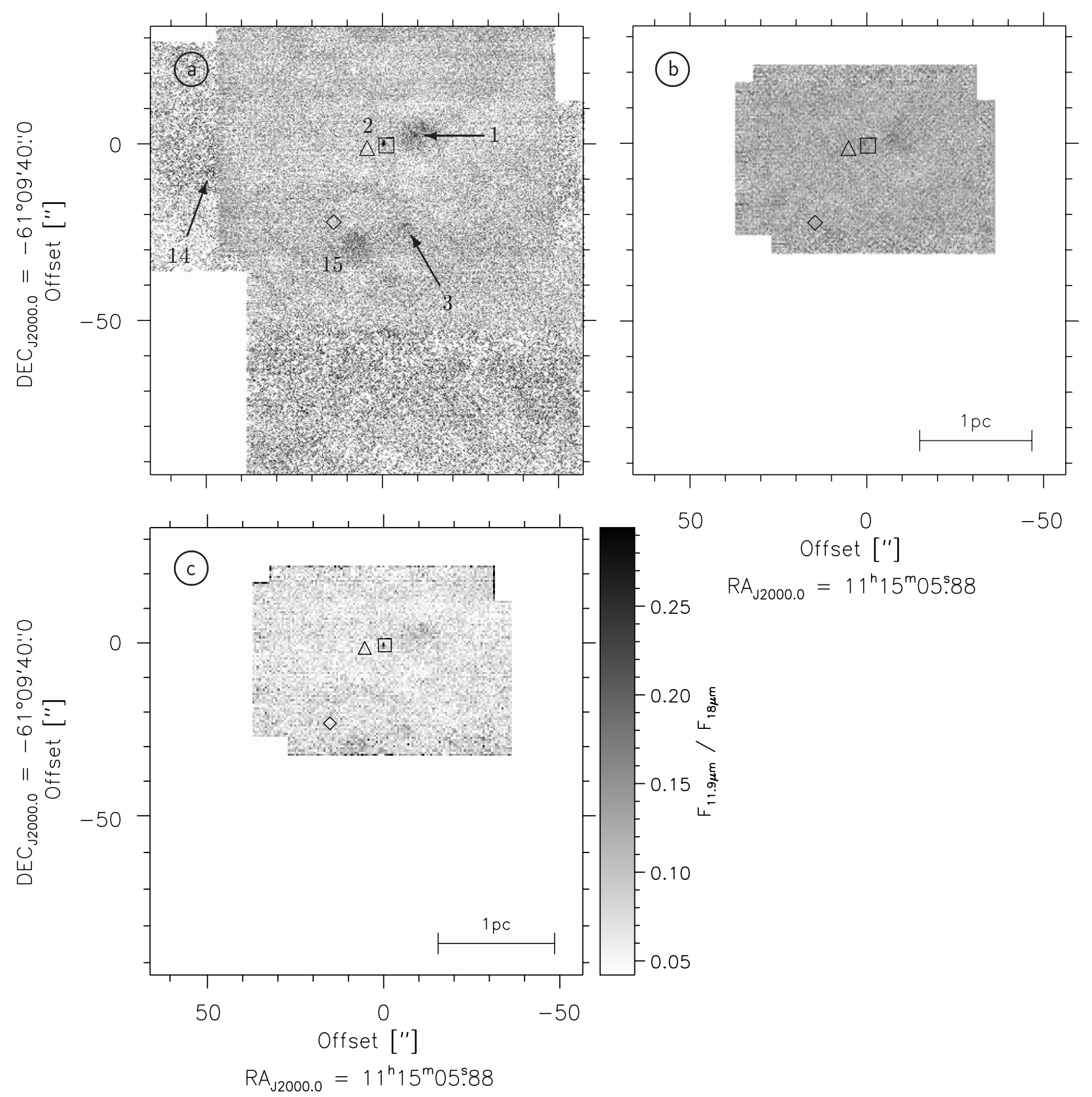

Fig. 5. Imaging results obtained for the northern field. Similar to Figs. $4 \mathrm{c}-\mathrm{e}$, the $11.9 \mu \mathrm{m}$ and $18 \mu \mathrm{m}$ data are presented in panels a) and b), respectively, while panel c) shows a map of the calibrated flux ratio.

$\mathrm{H} \alpha+[\mathrm{S} \mathrm{II}]+8160 \AA$ continuum data from the WFI at the ESO/MPG 2.2-m telescope (see Fig. 1), panel b displays $K_{\mathrm{s}}$ data obtained with ISAAC at the VLT Antu (see Nürnberger $\&$ Petr-Gotzens 2002). The corresponding $11.9 \mu \mathrm{m}$ and $18 \mu \mathrm{m}$ TIMMI 2 images are presented in panels $\mathrm{c}$ and d (with symbols as described above). Additionally, panel e gives the ratio of the calibrated $11.9 \mu \mathrm{m}$ and $18 \mu \mathrm{m}$ fluxes which is an indicator for the temperature of the emitting dust particles.

Finally, the survey results for the northern fields 11.9-2 and 18-2 are presented in Fig. 5. Similar to Figs. 4c-e, the $11.9 \mu \mathrm{m}$ and $18 \mu \mathrm{m}$ data are displayed in panels a and $\mathrm{b}$, respectively, while panel c shows a map of the calibrated flux ratio.

In total, 36 point sources are unambiguously detected at $11.9 \mu \mathrm{m}$. Thereof, some (very) faint sources - labeled
6 A-6R; see Fig. 6 - are located within the central $1^{\prime}$ of the OB cluster, with fluxes close to the nominal rms noise level of our data. However, the frames processed using the Bertero et al. method provide a significantly better signal-to-noise ratio than those obtained with the "nulling" technique, for two reasons. On the one hand, the Bertero et al. algorithm tends to improve the signal-to-noise ratio by itself as it amplifies real sources more efficiently than noise. On the other hand, the frequent rebinning of the data smoothes out the noise (at the expense of angular resolution). As a further test we constructed shift-and-add frames of the cluster center field and made sure that the faint sources were also visible on those frames and not due to any restoration artefacts (the negative source images were first shifted to the positive image positions and both 
Table 2. Detected point sources and diffuse emission knots.

\begin{tabular}{|c|c|c|c|c|c|c|c|c|}
\hline $\begin{array}{l}\text { source } \\
\text { NS \# }\end{array}$ & $\begin{array}{c}\text { counterpart }{ }^{\text {[a] }} \\
\text { (likely) }\end{array}$ & $\begin{array}{c}\Delta \mathrm{RA}^{[\mathrm{b}]} \\
{\left[{ }^{\prime \prime}\right]}\end{array}$ & $\begin{array}{c}\Delta \operatorname{Dec}^{[\mathrm{b}]} \\
\left.{ }^{\prime \prime}\right]\end{array}$ & $\begin{array}{c}\mathrm{RA}^{[\mathrm{c}]} \\
(\mathrm{J} 2000.0)\end{array}$ & $\begin{array}{c}\mathrm{DEC}^{[\mathrm{c}]} \\
(\mathrm{J} 2000.0)\end{array}$ & $\begin{array}{c}F_{11.9 \mu \mathrm{m}}{ }^{[\mathrm{d}]} \\
{[\mathrm{Jy}]}\end{array}$ & $\begin{array}{c}F_{18 \mu \mathrm{m}}{ }^{[\mathrm{d}]} \\
{[\mathrm{Jy}]}\end{array}$ & references $[\mathrm{e}]$ \\
\hline \multicolumn{9}{|c|}{ point sources } \\
\hline 1 & & $-8.7^{[\mathrm{a}]}$ & $2.4^{[\mathrm{a}]}$ & $11: 15: 04.67$ & $-61: 09: 37.6$ & 0.03 & 0.13 & \\
\hline 2 & $\mathrm{H}_{2} \mathrm{O} 291.58-0.43 / \mathrm{OH} 291.57-0.43$ & $0.0^{[\mathrm{a}]}$ & $-0.0^{[\mathrm{a}]}$ & $11: 15: 05.88$ & $-61: 09: 40.0$ & 0.09 & 0.25 & {$[1],[2],[3]$} \\
\hline 3 & & $-6.3^{[\mathrm{a}]}$ & $-23.2^{[\mathrm{a}]}$ & $11: 15: 05.00$ & $-61: 10: 03.2$ & 0.02 & 0.07 & \\
\hline 4 & IRS 4 & -54.5 & 142.8 & $11: 15: 03.79$ & $-61: 14: 22.4$ & 5.16 & & {$[4]$} \\
\hline $5 \mathrm{~A}$ & & -52.4 & 97.5 & $11: 15: 04.07$ & $-61: 15: 07.7$ & 2.10 & & \\
\hline $5 \mathrm{~B}$ & (J111504.6-611511) & -48.7 & 91.1 & $11: 15: 04.59$ & $-61: 15: 14.1$ & 0.72 & & {$[5]$} \\
\hline $5 \mathrm{C}$ & (J111505.3-611516) & -42.8 & 86.2 & $11: 15: 05.40$ & $-61: 15: 19.0$ & 0.16 & & {$[5]$} \\
\hline $6 \mathrm{~A}$ & MDS 30,31,26 / MTT A1-A3 & -26.5 & 67.3 & $11: 15: 07.67$ & $-61: 15: 37.9$ & 0.19 & & {$[6],[7],[8],[9]$} \\
\hline $6 B$ & MDS 23 / MTT B & -25.2 & 67.3 & $11: 15: 07.85$ & $-61: 15: 37.9$ & 0.13 & & {$[6],[7],[8],[9]$} \\
\hline $6 \mathrm{D}$ & MDS 49,50,52,53 / MTT D & -28.8 & 67.3 & $11: 15: 07.35$ & $-61: 15: 37.9$ & 0.02 & & {$[6],[7]$} \\
\hline $6 \mathrm{~L}$ & & -17.9 & 62.5 & $11: 15: 08.86$ & $-61: 15: 42.6$ & $(0.01)$ & & \\
\hline $6 \mathrm{P}$ & & -19.1 & 53.6 & $11: 15: 08.70$ & $-61: 15: 51.5$ & $(0.01)$ & & \\
\hline 7 & MTT 64 & -52.7 & 58.6 & $11: 15: 04.03$ & $-61: 15: 46.6$ & 1.22 & & [7] \\
\hline 8 & MTT 120 & -39.2 & 58.6 & $11: 15: 05.90$ & $-61: 15: 46.6$ & 0.62 & & [7] \\
\hline $9 \mathrm{~A}$ & IRS 9A & 0.0 & -0.0 & $11: 15: 11.34$ & $-61: 16: 45.2$ & 21.88 & 51.69 & {$[10]$} \\
\hline $9 \mathrm{~B}$ & IRS 9B & -5.2 & 4.1 & $11: 15: 10.62$ & $-61: 16: 41.1$ & 0.89 & 1.58 & [11] \\
\hline $9 \mathrm{C}$ & IRS 9C & -3.5 & 0.3 & $11: 15: 10.86$ & $-61: 16: 44.9$ & 0.88 & 1.23 & [11] \\
\hline 10 & $\mathrm{H}_{2} \mathrm{O} 291.64-0.55$ & 27.3 & -50.9 & $11: 15: 15.13$ & $-61: 17: 36.1$ & 0.12 & & {$[2]$} \\
\hline 11 & & -8.7 & -53.1 & $11: 15: 10.13$ & $-61: 17: 38.3$ & 0.29 & & \\
\hline 12 & & -34.9 & -71.7 & $11: 15: 06.50$ & $-61: 17: 56.8$ & 0.30 & & \\
\hline $13 \mathrm{~A}$ & $\mathrm{H}_{2} \mathrm{O} 291.65-0.60$ & 0.0 & -220.0 & $11: 15: 11.34$ & $-61: 20: 25.1$ & 0.38 & & {$[2]$} \\
\hline $13 \mathrm{~B}$ & & -0.9 & -231.6 & $11: 15: 11.22$ & $-61: 20: 36.7$ & 0.29 & & \\
\hline $13 \mathrm{C}$ & & -5.2 & -243.8 & $11: 15: 10.61$ & $-61: 20: 48.9$ & 0.21 & & \\
\hline \multicolumn{9}{|c|}{ diffuse emission knots } \\
\hline 14 & & $48.2^{[\mathrm{a}]}$ & $-8.1^{[\mathrm{a}]}$ & $11: 15: 12.54$ & $-61: 09: 48.1$ & 0.02 & & \\
\hline 15 & $\mathrm{CH}_{3} \mathrm{OH} 291.58-0.43$ & $8.1^{[\mathrm{a}]}$ & $-28.9^{[\mathrm{a}]}$ & $11: 15: 07.00$ & $-61: 10: 08.9$ & 0.02 & 0.17 & {$[12]$} \\
\hline $16 \mathrm{~A}$ & & -55.3 & 92.9 & $11: 15: 03.66$ & $-61: 15: 12.3$ & 0.37 & & \\
\hline $16 \mathrm{~B}$ & & -54.2 & 84.4 & $11: 15: 03.83$ & $-61: 15: 20.7$ & 0.12 & & \\
\hline $17 \mathrm{~A}$ & ring nebula around Sher 25 & -27.3 & 91.1 & $11: 15: 07.55$ & $-61: 15: 14.1$ & 0.15 & & {$[13],[14]$} \\
\hline $17 \mathrm{~B}$ & ring nebula around Sher 25 & -20.7 & 83.6 & $11: 15: 08.47$ & $-61: 15: 21.6$ & 0.19 & & {$[13],[14]$} \\
\hline 18 & & -86.5 & 72.0 & $11: 14: 59.35$ & $-61: 15: 33.2$ & 0.20 & & \\
\hline 19 & & -59.4 & 71.1 & $11: 15: 03.10$ & $-61: 15: 34.1$ & 0.16 & & \\
\hline 20 & IRS 14 & -60.0 & 60.6 & $11: 15: 03.02$ & $-61: 15: 44.5$ & 0.58 & & {$[4]$} \\
\hline 21 & & -71.3 & 49.9 & $11: 15: 01.44$ & $-61: 15: 55.3$ & 0.22 & & \\
\hline 22 & & -41.4 & 49.0 & $11: 15: 05.60$ & $-61: 15: 56.1$ & 0.09 & & \\
\hline 23 & (IRS 1) & -60.3 & 48.5 & $11: 15: 02.98$ & $-61: 15: 56.7$ & 0.63 & & {$[4]$} \\
\hline 24 & $\mathrm{H}_{2} \mathrm{O} 291.61-0.53$ & -51.6 & 45.3 & $11: 15: 04.19$ & $-61: 15: 59.9$ & 1.26 & 4.34 & [2] \\
\hline 25 & & -2.3 & 42.9 & $11: 15: 11.02$ & $-61: 16: 02.2$ & 0.26 & & \\
\hline $26 \mathrm{~A}$ & & -26.5 & 42.8 & $11: 15: 07.67$ & $-61: 16: 02.4$ & 0.05 & & \\
\hline 27 & IRS 8 & -11.3 & 42.6 & $11: 15: 09.77$ & $-61: 16: 02.5$ & 0.06 & & {$[4]$} \\
\hline 28 & & 34.3 & 39.7 & $11: 15: 16.10$ & $-61: 16: 05.4$ & 0.21 & & \\
\hline 29 & & 11.3 & 35.4 & $11: 15: 12.91$ & $-61: 16: 09.8$ & 0.68 & & \\
\hline 30 & & -44.4 & 24.1 & $11: 15: 05.19$ & $-61: 16: 21.1$ & 0.09 & & \\
\hline 31 & & -60.3 & 15.9 & $11: 15: 02.98$ & $-61: 16: 29.2$ & 0.48 & & \\
\hline $32 \mathrm{~A}$ & (J111505.3-611638) & -40.8 & 6.4 & $11: 15: 05.68$ & $-61: 16: 38.8$ & 0.67 & 1.74 & {$[5]$} \\
\hline $32 \mathrm{~B}$ & & -33.8 & 5.8 & $11: 15: 06.65$ & $-61: 16: 39.4$ & 0.48 & & \\
\hline 33 & & -56.8 & 4.2 & $11: 15: 03.46$ & $-61: 16: 41.0$ & 0.60 & & \\
\hline 34 & IRS 2 & -14.6 & -5.8 & $11: 15: 09.32$ & $-61: 16: 51.0$ & 0.20 & & [4] \\
\hline $35 \mathrm{~A}$ & MTT 58 & -27.6 & -11.0 & $11: 15: 07.51$ & $-61: 16: 56.2$ & 0.28 & 0.58 & [7] \\
\hline $35 \mathrm{~B}$ & & -31.7 & -17.4 & $11: 15: 06.94$ & $-61: 17: 02.6$ & 0.20 & & \\
\hline 36 & & 25.0 & -14.8 & $11: 15: 14.81$ & $-61: 17: 00.0$ & 0.43 & 0.74 & \\
\hline 37 & MM 2 & 3.8 & -15.4 & $11: 15: 11.87$ & $-61: 17: 00.6$ & 0.68 & 1.79 & {$[15]$} \\
\hline 38 & & -49.2 & -15.4 & $11: 15: 04.51$ & $-61: 17: 00.6$ & 0.18 & & \\
\hline $39 \mathrm{~A}$ & & -10.2 & -17.4 & $11: 15: 09.93$ & $-61: 17: 02.6$ & 0.43 & & \\
\hline $39 B$ & & -12.5 & -20.6 & 11:15:09.61 & $-61: 17: 05.8$ & 0.23 & & \\
\hline 40 & & -42.8 & -22.6 & 11:15:05.40 & $-61: 17: 07.8$ & 0.21 & & \\
\hline 41 & & -55.9 & -29.0 & $11: 15: 03.58$ & $-61: 17: 14.2$ & 0.15 & & \\
\hline 42 & & -35.5 & -30.7 & 11:15:06.41 & $-61: 17: 15.9$ & 0.20 & & \\
\hline 43 & & -14.6 & -32.5 & $11: 15: 09.32$ & $-61: 17: 17.7$ & 0.12 & & \\
\hline 44 & & -21.2 & -36.8 & 11:15:08.39 & $-61: 17: 22.0$ & 0.09 & & \\
\hline $45 \mathrm{~A}$ & & -27.3 & -40.6 & $11: 15: 07.55$ & $-61: 17: 25.8$ & 0.27 & & \\
\hline $45 \mathrm{~B}$ & & -22.6 & -42.4 & $11: 15: 08.21$ & $-61: 17: 27.5$ & 0.19 & & \\
\hline 46 & & -45.2 & -96.6 & 11:15:05.08 & $-61: 18: 21.8$ & 0.19 & & \\
\hline 47 & (OH 291.64-00.56) & 23.7 & -101.5 & $11: 15: 14.63$ & $-61: 18: 26.7$ & 0.12 & & [16] \\
\hline 48 & & -17.3 & -256.5 & 11:15:08.94 & $-61: 21: 01.7$ & 0.21 & & \\
\hline
\end{tabular}


Table 2. Continued.

\begin{tabular}{|c|c|c|c|c|c|c|c|c|}
\hline $\begin{array}{l}\text { source } \\
\text { NS \# }\end{array}$ & $\begin{array}{c}\text { counterpart }^{[\mathrm{a}]} \\
\text { (likely) }^{-} \\
\end{array}$ & $\begin{array}{c}\Delta \mathrm{RA}^{[\mathrm{b}]} \\
{\left[{ }^{\prime \prime}\right]} \\
\end{array}$ & $\begin{array}{c}\Delta \operatorname{Dec}^{[b]} \\
{\left[{ }^{\prime \prime}\right]}\end{array}$ & $\begin{array}{c}\mathrm{RA}^{[\mathrm{c}]} \\
(\mathrm{J} 2000.0)\end{array}$ & $\begin{array}{c}\mathrm{DEC}^{[\mathrm{c}]} \\
(\mathrm{J} 2000.0)\end{array}$ & $\begin{array}{c}F_{11.9 \mu \mathrm{m}}{ }^{[\mathrm{d}]} \\
{[\mathrm{Jy}]} \\
\end{array}$ & $\begin{array}{c}F_{18 \mu \mathrm{m}}{ }^{[\mathrm{d}]} \\
{[\mathrm{Jy}]} \\
\end{array}$ & references $^{[\mathrm{e}]}$ \\
\hline \multicolumn{9}{|c|}{ additional point sources in the cluster center (only detected with the Bertero et al. method) } \\
\hline $6 \mathrm{C}$ & MDS 18 / MTT C & -24.5 & 67.5 & 11:15:07.94 & $-61: 15: 37.6$ & 0.01 & & {$[6],[7],[8],[9]$} \\
\hline $6 \mathrm{E}$ & (MDS 60) & -30.0 & 64.3 & 11:15:07.18 & $-61: 15: 40.9$ & 0.01 & & [6] \\
\hline $6 \mathrm{~F}$ & & $(-27.7)$ & $(67.4)$ & $(11: 15: 07.50)$ & $(-61: 15: 37.8)$ & $(0.01)$ & & \\
\hline 6G & $($ MDS 68,69) & -22.1 & 64.7 & $11: 15: 08.28$ & $-61: 15: 40.4$ & 0.01 & & [6] \\
\hline $6 \mathrm{H}$ & & $(-28.5)$ & $(84.8)$ & $(11: 15: 07.39)$ & $(-61: 15: 20.4)$ & $(0.01)$ & & \\
\hline $6 \mathrm{I}$ & & -35.0 & 77.3 & $11: 15: 06.49$ & $-61: 15: 27.9$ & 0.01 & & \\
\hline $6 \mathrm{~J}$ & & $(-28.3)$ & $(75.9)$ & $(11: 15: 07.42)$ & $(-61: 15: 29.3)$ & $(0.01)$ & & \\
\hline $6 \mathrm{~K}$ & & -16.1 & 66.6 & $11: 15: 09.10$ & $-61: 15: 38.6$ & 0.01 & & \\
\hline $6 \mathrm{M}$ & (MDS 70) & -24.3 & 61.0 & 11:15:07.97 & $-61: 15: 44.1$ & 0.01 & & [6] \\
\hline $6 \mathrm{~N}$ & (MDS 58 / MTT 15B) & -30.5 & 60.1 & $11: 15: 07.12$ & $-61: 15: 45.1$ & 0.01 & & {$[6],[7]$} \\
\hline 60 & & -32.9 & 57.3 & $11: 15: 06.78$ & $-61: 15: 47.9$ & 0.01 & & \\
\hline $6 \mathrm{Q}$ & & (6.5) & $(20.2)$ & $11: 15: 12.25$ & $-61: 16: 25.0$ & $(0.01)$ & & \\
\hline $6 \mathrm{R}$ & & 33.7 & 17.9 & 11:15:16.01 & $-61: 16: 27.3$ & 0.02 & & \\
\hline \multicolumn{9}{|c|}{ additional diffuse emission knots in the cluster center (only detected with the Bertero et al. method) } \\
\hline 26B & & -23.6 & 39.7 & 11:15:08.07 & $-61: 16: 05.5$ & 0.01 & & \\
\hline
\end{tabular}

Notes: [a] "Likely" means close-by but located outside of the $3 \sigma$ position error margins; [b] all offsets are given relative to source NS 9A (IRS 9A), except for sources NS 1, NS 2, NS 3, NS 14 and NS 15 (i.e. sources located in the northern fields) for which offsets are given relative to source NS 2; [c] for the point sources: $\sigma_{\mathrm{RA}} \sim \pm 0.04, \sigma_{\mathrm{DEC}} \sim \pm 0$ ". 3 ; for the diffuse emission knots the positions refer to the emission peak and should be considered by a factor of 2-3 less accurate; [d] rms noise values are $\sigma_{11.9 \mu \mathrm{m}} \sim \pm 0.01 \mathrm{Jy}$, and $\sigma_{18 \mu \mathrm{m}} \sim \pm 0.02 \mathrm{Jy}$ for the northern fields, $\sigma_{11.9 \mu \mathrm{m}} \sim \pm 0.01 \mathrm{Jy}$ and $\sigma_{18 \mu \mathrm{m}} \sim \pm 0.03 \mathrm{Jy}$ for the southern fields; [e] reference for the position of the (likely) counterpart; [1] Braz \& Scalise (1982); [2] Caswell et al. (1989); [3] Caswell (1998); [4] Frogel et al. (1977); [5] Tapia et al. (2001); [6] Moffat et al. (1994); [7] Melnick et al. (1989); [8] van den Bos (1928); [9] Hofmann \& Weigelt (1986); [10] Nürnberger \& Petr-Gotzens (2002); [11] Nürnberger (2003); [12] Caswell et al. (1995); [13] Brandner et al. (1997a); [14] Brandner et al. (1997b); [15] Nürnberger et al. (2002b); [16] Braz \& Epchtein (1983).

images then added together, providing full angular resolution and best point source sensitivity at the expense of messing up all extended structures). We made also sure that the faint sources were not due to any "noisy pixels" in an individual frame. Alltogether, this makes us confident to deal with real sources and not with noise, but higher sensitivity observations definitely remain desirable.

Furthermore, we have identified 42 knot-like emission features, most of which apparently do not have any stellar counterpart at either optical or near IR wavelengths. At least 7 filamentary features are found to be well correlated with similar structures of highly ionized material observed in the optical and/or near IR. Also the heads of the two prominent pillars are clearly seen: while the head of the eastern one is seen in absorption against the bright mid IR background, the head of the western one exhibits several bright rims of compressed gas and dust. In the $18 \mu \mathrm{m}$ data we have identified 6 point sources and 6 knots of diffuse emission. The coordinates and fluxes of all detected point sources and diffuse emission knots are listed in Table 2.

\section{Discussion of individual sources}

In the following sections we discuss the nature of the detected mid IR sources and provide some relevant background information. For that purpose we have converted the measured $11.9 \mu \mathrm{m}$ and $18 \mu \mathrm{m}$ fluxes into apparent magnitudes. In addition, we have searched the literature for corresponding
$J H K$ magnitudes in order to better characterize our sources. As far as near IR data are available in the literature, the apparent $J H K$ magnitudes of our sources are listed in Table 3 along with their $N$ and $Q$ band magnitudes derived from the TIMMI 2 data.

With these values at hand, we calculated the $(J-K)$ and $(K-N)$ colours as well as the absolute $K$ band magnitudes. Figure 7 shows the resulting $(J-K)-(K-N)$ and $M_{K}-(K-N)$ diagrams. Both plots demonstrate the large diversity of mid IR sources found within the NGC 3603 region and clearly reveal the large mid IR excesses of some of them. The faint $11.9 \mu \mathrm{m}$ sources found in the cluster center are rather luminous in the near IR $(6 \mathrm{~A}-6 \mathrm{G})$ and tend to have a weak - but nevertheless noticable - mid IR excess. They are located close to the loci of giants and supergiants, which are represented by the moderately thick and thin straight lines. In a similar way, the loci of main sequence stars are indicated by the thick lines, following Koornneef (1983a,b) and Ducati et al. (2001).

We emphasize that in both diagrams of Fig. 7 we have corrected for $A_{\mathrm{V}}=4.5$, i.e. the foreground extinction measured along the line-of-sight towards the OB cluster. Considering the reddening vectors for interstellar extinction, it appears very unlikely that sources with extremely red colours (e.g. $9 \mathrm{~A}-\mathrm{C}, 5 \mathrm{~A}-\mathrm{C}$ ) and well separated from the main sequence are highly reddened, high mass background sources. Instead, they may constitute a population of relatively young sources within NGC 3603, which are still in a pre-main sequence phase of their evolution. In order to further constrain their nature we 


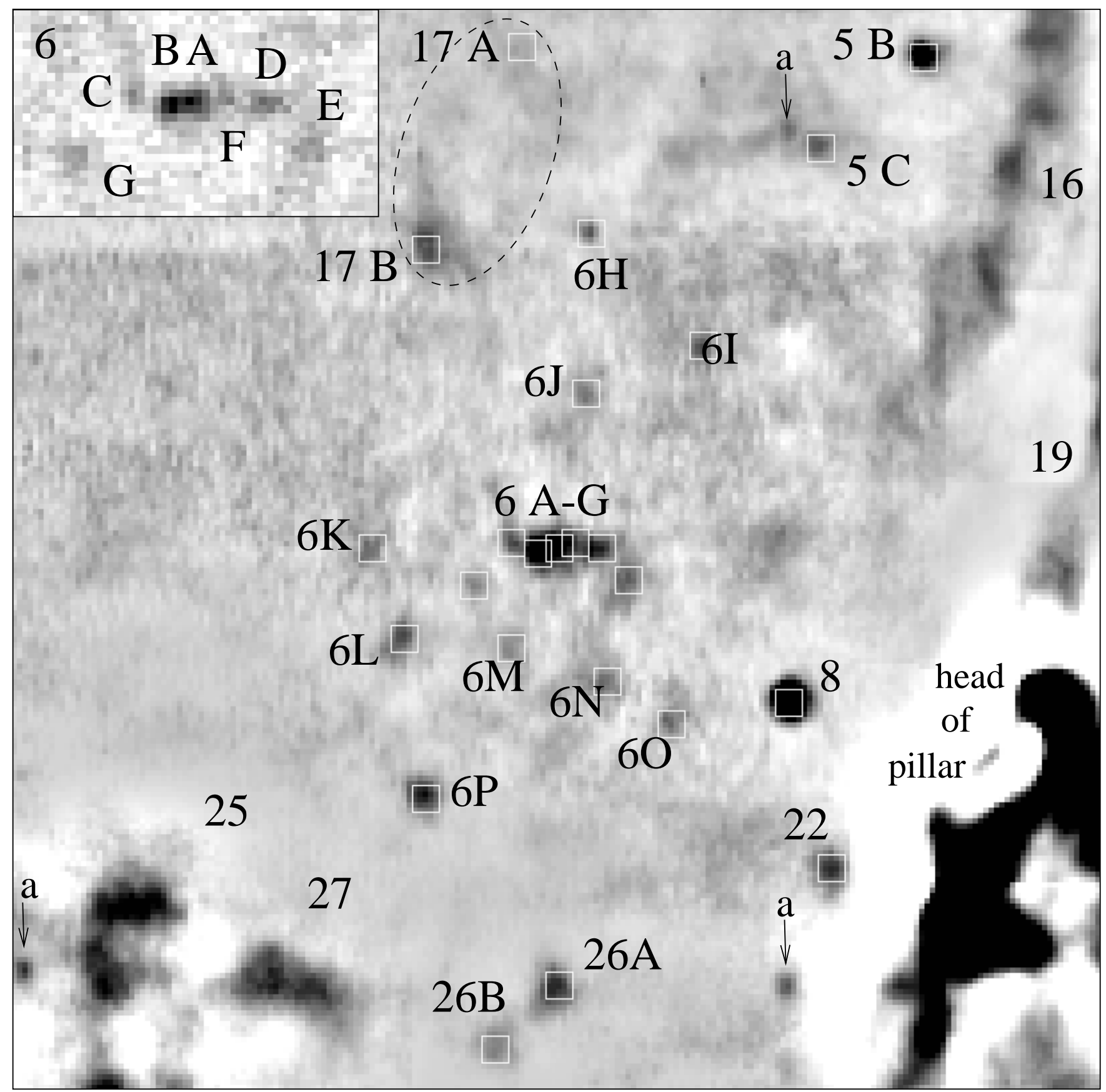

Fig. 6. Finding chart (field-of-view is $1^{\prime} \times 1^{\prime}$ ) for the weak $11.9 \mu$ m sources detected in the OB cluster, using the restoration method of Bertero et al. (2000; see Sect. 3 for details). The insert in the upper left corner shows a zoom-in of the very center of HD 97950 which was obtained via the classical shift-and-add technique, preserving the angular resolution. The dashed ellipse outlines the ring-like emission feature seen on optical images around Sher 25 (Brandner et al. 1997a,b). Artefacts of the image restoration are marked by "a".

calculated the spectral index $\alpha_{\mathrm{IR}}=\mathrm{d} \log \left(\lambda \mathrm{F}_{\lambda}\right) / \mathrm{d} \log \lambda$ between $\lambda=2.2 \mu \mathrm{m}$ and $11.9 \mu \mathrm{m}$ (see Col. 7 of Table 3), which gives a good measure of the mid IR excess usually attributed to the presence of dust in a circumstellar envelope/ disk.

We compare the obtained $\alpha_{\mathrm{IR}}$ values with the evolutionary classification scheme of (low mass) young stellar objects originally introduced by Lada (1987) and subsequently revised by André \& Montmerle (1994). This scheme predicts a progressive decrease of the amount of circumstellar material with stellar age, from IR class $0\left(\sim 10^{4} \mathrm{yr} ; \alpha_{\mathrm{IR}}>0\right)$ to IR class III $\left(\sim 10^{7} \mathrm{yr} ; \alpha_{\mathrm{IR}}<-1.5\right)$. Assuming that similar classification criteria hold for intermediate and high mass stars, the $\alpha_{\mathrm{IR}}$ values of our sources support the picture already derived above from the $(J-K)-(K-N)$ and $M_{K}-(K-N)$ diagrams, namely that (in most cases) we are dealing with young stellar objects surrounded by warm dust. 
Table 3. Measured apparent magnitudes (without applying any correction for foreground extinction) and IR indices of the sources discussed in Sect. 6.

\begin{tabular}{ccccccrr}
\hline \hline $\begin{array}{c}\text { source } \\
\text { NS \# }\end{array}$ & $\begin{array}{c}J \\
{[\mathrm{mag}]}\end{array}$ & $\begin{array}{c}H \\
{[\mathrm{mag}]}\end{array}$ & $\begin{array}{c}K \\
{[\mathrm{mag}]}\end{array}$ & $\begin{array}{c}N 11.9 \\
{[\mathrm{mag}]}\end{array}$ & $\begin{array}{c}Q 1 \\
{[\mathrm{mag}]}\end{array}$ & $\alpha_{\mathrm{IR}}{ }^{[\mathrm{a}]}$ & references ${ }^{[\mathrm{b}]}$ \\
\hline 4 & 6.73 & 5.10 & 4.39 & 2.11 & & -1.63 & {$[1]$} \\
5A & & & 14.66 & 3.09 & & 3.43 & {$[2]$} \\
5B & 13.14 & 12.09 & 10.98 & 4.24 & & 0.80 & {$[3]$} \\
5C & 13.93 & 12.62 & 11.56 & 5.87 & & 0.23 & {$[3]$} \\
6A & 8.58 & 8.28 & 7.68 & 5.78 & & -1.84 & {$[4]$} \\
6B & 8.53 & 8.16 & 7.65 & 6.06 & & -2.01 & {$[4]$} \\
6C & 9.23 & 8.81 & 8.46 & 8.89 & & -3.11 & {$[4]$} \\
6D & 10.64 & 10.26 & 9.98 & 8.45 & & -2.04 & {$[4]$} \\
6E & 11.46 & 11.13 & 10.89 & 8.89 & & -1.79 & {$[4]$} \\
6G & 12.18 & 11.87 & 11.66 & 8.89 & & -1.37 & {$[4]$} \\
6N & 11.56 & 11.20 & 10.89 & 8.89 & & -1.79 & {$[4]$} \\
7 & 12.02 & 11.28 & 11.29 & 3.67 & & 1.28 & {$[3]$} \\
8 & 12.37 & 10.02 & 8.58 & 4.41 & & -0.60 & {$[3]$} \\
9A & 12.87 & 10.92 & 8.55 & 0.54 & -1.85 & 1.49 & {$[5]$} \\
9B & 14.63 & 13.94 & 12.23 & 4.02 & 1.94 & 1.60 & {$[5]$} \\
9C & 16.71 & 15.34 & 11.85 & 4.03 & 2.21 & 1.39 & {$[5]$} \\
10 & & & 10.82 & 6.23 & & -0.37 & {$[2]$} \\
11 & & & 13.38 & 5.25 & & 1.56 & {$[2]$} \\
12 & & & 14.37 & 5.19 & & 2.13 & {$[2]$} \\
13B & & & 16.83 & 5.23 & & 3.45 & {$[2]$} \\
32A & 14.20 & 14.14 & 12.38 & 4.32 & 1.83 & 1.52 & {$[3]$} \\
34 & 14.13 & 12.77 & 11.48 & 5.64 & & 0.31 & {$[3]$} \\
\hline
\end{tabular}

Notes: [a] Spectral index as measured between $2.2 \mu \mathrm{m}$ and $11.9 \mu \mathrm{m}$ (see the text for details); [b] reference for the $J H K$ magnitudes; [1] Frogel et al. (1977); [2] Nürnberger, unpublished; [3] Tapia et al. (2001); [4] Eisenhauer et al. (1998); [5] Nürnberger (2003).

\subsection{IRS 9 - an association of protostars}

Both at $11.9 \mu \mathrm{m}$ and at $18 \mu \mathrm{m}$ NS 9A alias IRS 9A clearly stands out as the most luminous mid IR source of NGC 3603, with fluxes of $F_{11.9 \mu \mathrm{m}}=21.88 \mathrm{Jy}$ and $F_{18 \mu \mathrm{m}}=51.69 \mathrm{Jy}$, respectively. In its immediate vicinity two more point sources are clearly detected: NS 9B alias IRS 9B $\left(F_{11.9 \mu \mathrm{m}}=0.89 \mathrm{Jy}\right.$, $\left.F_{18 \mu \mathrm{m}}=1.58 \mathrm{Jy}\right)$ and NS 9C alias IRS 9C $\left(F_{11.9 \mu \mathrm{m}}=0.88 \mathrm{Jy}\right.$, $\left.F_{18 \mu \mathrm{m}}=1.23 \mathrm{Jy}\right)$. These flux values suggest the existence of large amounts of gas and dust in the circumstellar environments (shells and/or disks) of all three sources, which - together with their steeply rising spectral energy distributions from near to mid IR wavelengths - indicates early evolutionary stages $\left(<10^{5} \mathrm{yr}\right.$; Nürnberger et al. 2002a).

Indeed, the close spatial arrangement of IRS 9A, 9B and 9C supports the hypothesis that IRS 9 represents an association of protostars in its own right. Within a radius of $15^{\prime \prime}$ around IRS 9A Tapia et al. (2001) report a total of at least 12 sources with considerable near IR excess emission. The fact that only three - four, if we also include NS $34=$ IRS 2 - members of this association are seen at mid IR wavelengths, might be explained by the (in comparison to todays near IR capabilities still very) limited sensitivity of the TIMMI 2 camera (Nürnberger et al. 2002a).

\subsection{Young sources with $K$ excess or maser emission}

Despite of this restriction, however, some more $11.9 \mu \mathrm{m}$ sources (either point-like or diffuse) correlate quite well in position (typically within $1^{\prime \prime}-3^{\prime \prime}$ ) with near IR sources of spectral type B showing large $K$-band excesses (Tapia et al. 2001): NS 5B, NS 5C, NS 7, NS 8, NS 20 and NS 32A. In the neighbourhood of NS 10 even three prominent $K$-band excesses sources, a maser source $\left(\mathrm{H}_{2} \mathrm{O} 291.64-0.55\right.$; Caswell et al. 1989) and a peak of 3.4-cm radio emission (De Pree et al. 1999) are known. Also NS 11 and NS 12 have moderately bright counterparts in the $K$-band, but do not exhibit significant $K$-band excess emission.

Probably, as already suggested by Tapia et al. (2001), all of them constitute a population of (slightly) younger stars than those found towards the very center of the OB cluster. The detection of mid IR emission from these sources for itself implies fluxes in excess of those expected from photospheric emission of OB stars in NGC 3603 (note that even the brightest stars in the cluster are only detected at $11.9 \mu \mathrm{m}$ because of excess emission; see Sect. 6.6). Whereas the compact $N$-band sources likely indicate circumstellar dust disks or shells, the more extended features (which often are a bit offset from the stellar counterparts) are probably arising from photon-dominated regions (PDRs) in small $\mathrm{H}_{\mathrm{II}}$ regions associated with the stars.

Taking into account their positions relative to HD 97950 (e.g. IRS 9 has a projected distance of about $70^{\prime \prime}$ ) and also considering the redefined radius of the OB cluster $\left(\sim 150^{\prime \prime}\right.$; Nürnberger \& Petr-Gotzens 2002), these young stars formed well within the cluster boundaries. But star formation in NGC 3603 is not at all restricted to the cluster alone, as we find several mid IR sources outside the cluster, too. At the 

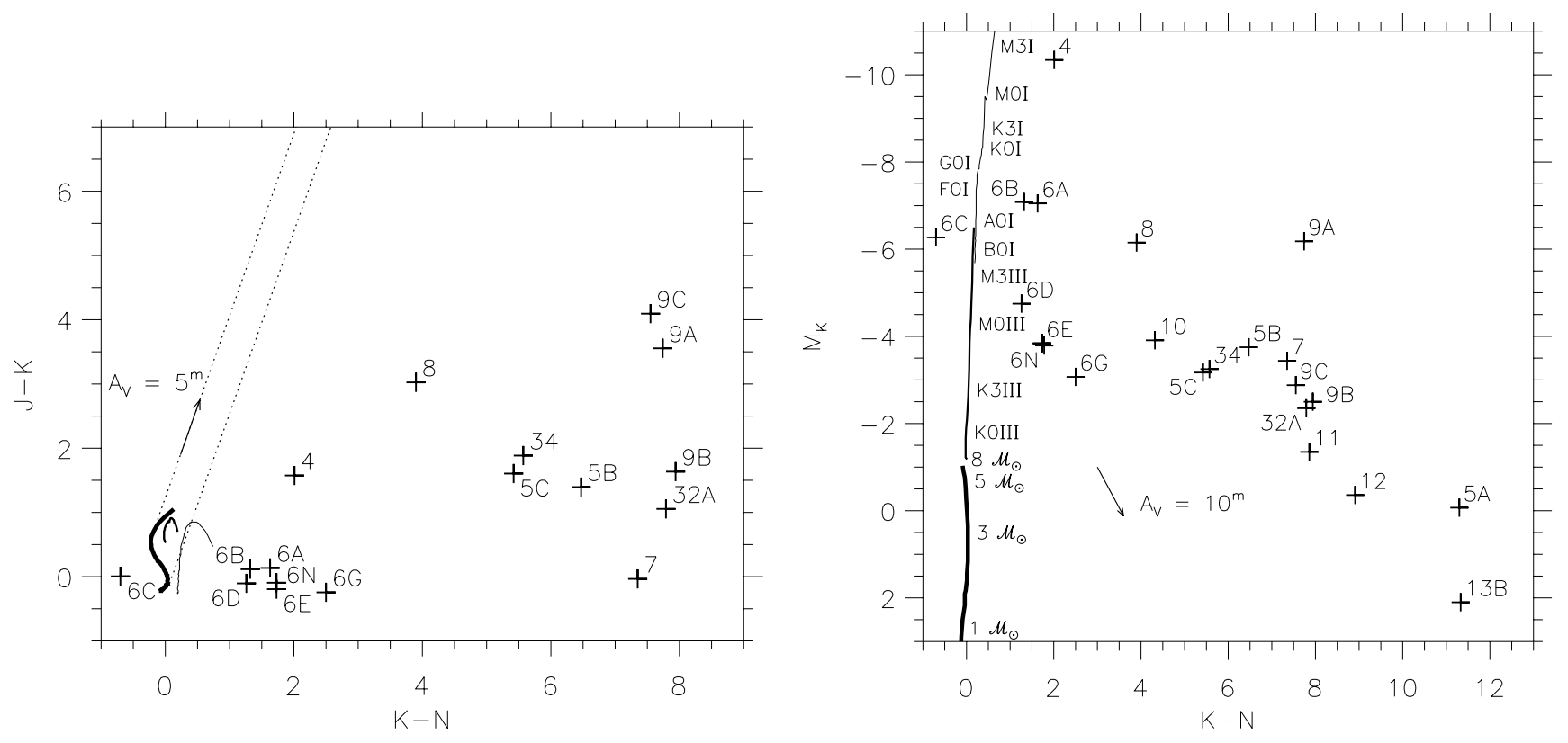

Fig. 7. Near/mid IR colour-magnitude and two-colour diagrams of the sources discussed in Sect. 6. Both diagrams are corrected for the foreground extinction of $A_{\mathrm{V}}=4 \mathrm{~m} 5$. The straight lines (from thick to thin) indicate the loci of main sequence stars, giants and supergiants (Koornneef 1983a, 1983b; Ducati et al. 2001).

southern end of our survey field 11.9-1 we discover three point sources (NS 13A-C) which are associated with the molecular clump MM 4 (Nürnberger et al. 2002b) and the water maser $\mathrm{H}_{2} \mathrm{O} 291.65-0.60$ (Caswell et al. 1989). About 20-30" further southward a patch of extended mid IR emission is seen.

Also in the northern field 11.9-2 we detect three faint point sources. Located about $6^{\prime}$ away from HD 97950, the position of NS 2 is coincident with both water and hydroxyl masers $\left(\mathrm{H}_{2} \mathrm{O} 291.58-0.43\right.$ and $\mathrm{OH} 291.57-0.43$; Braz \& Scalise 1982; Caswell et al. 1989; Caswell 1998). NS 1, surrounded by a halo of extended emission, and NS 3 lie about $10^{\prime \prime}$ the the west and about $20^{\prime \prime}$ to the south, respectively. Additionally, a closeby knot of $11.9 \mu \mathrm{m}$ emission appears to be related to the methanol maser $\mathrm{CH}_{3} \mathrm{OH} 291.58-0.43$ (Caswell et al. 1995). All four northern mid IR sources are very likely associated with the massive molecular clump MM 11 (Nürnberger et al. 2002b), which strongly suggests that star formation is also going on in the far north of the OB cluster.

This, by itself, is an important result because the star formation process in NGC 3603 was so far supposed to have started in the north of the OB cluster and to proceed towards its south (Walborn 1973; Melnick et al. 1989). Together with the overall spatial distribution of the molecular gas (Nürnberger et al. 2002b) our mid IR data suggest a different scenario, with the $\mathrm{OB}$ cluster being placed right at the center of all the star formation processes going on within NGC 3603.

\subsection{IRS 1}

Proposed by Frogel et al. (1977) to be - together with IRS 2 the brightest source at mid IR wavelengths, no discrete bright point source is detected towards the position of IRS 1, neither in our $11.9 \mu \mathrm{m}$ data nor in our $18 \mu \mathrm{m}$ data. Given the large aperture of $14^{\prime \prime} .5$ used by Frogel et al. (1977), their mid IR fluxes listed for IRS 1 likely correspond to the bright, extended $11.9 / 18 \mu \mathrm{m}$ structure associated with the head of the northwestern pillar. Comparison of optical and near IR images of this region with the new mid IR data shows a very good correspondance between the nebular structures seen at individual wavelengths. Clearly, most of the mid IR emission in this area is thus due to warm dust in the highly structured ionization front visible in optical and near IR images. A bright protrusion (labeled as NS 7) of this complex mid IR emission may contain a compact source which is associated with the $K$-band excess B type star MTT 64 identified by Tapia et al. (2001). No other compact substructures in this pillar can be identified with other bright $K$-band sources.

\subsection{IRS 2}

IRS 2 has been suggested to represent the most luminous source of a new generation of stars forming towards the south of the NGC 3603 OB cluster, attributing the total mid IR flux detected by Frogel et al. (1977) to this source. Its position as obtained from its suggested $K$-band counterpart (Tapia et al. 2001) - is at the very tip of an opaque protrusion apparently extending from a larger filament of molecular gas towards the NGC 3603 cluster. However, the rim-like appearance of this filament (although clearly illuminated by the massive stars of the nearby cluster) is primarily due to absorption caused by a dense molecular clump (visible as a dark silhouette against the extended mid IR emission), which is located in the foreground and not at all associated with the NGC $3603 \mathrm{H}$ in region (Nürnberger et al. 2002b).

In perfect agreement with this explanation, our mid IR data suggest a greatly revised picture of IRS 2 . At its position we 
see only a very faint, compact $11.9 \mu$ m source (NS 34) against highly structured diffuse emission, and no source at $18 \mu \mathrm{m}$. Obviously, the mid IR brightness of this source has been strongly overestimated in the work of Frogel et al. (1977). IRS 2 might be a young B type star, but it is by far not as luminous as previously assumed. As it can be seen doubtless from our data, the source truly dominating the mid IR luminosity (regarding point sources) in the entire survey field is IRS 9A. Due to the lack of angular resolution, Frogel et al. (1977) were simply unable to separate and discriminate between IRS 2 and IRS 9A.

\subsection{IRS 4}

The second brightest source of our $11.9 \mu \mathrm{m}$ survey is NS 4 alias IRS $4\left(F_{11.9 \mu \mathrm{m}} \sim 5.16 \mathrm{Jy}\right)$, for which Frogel et al. (1977) give a slightly larger flux of $F_{10 \mu \mathrm{m}} \sim 9.1 \mathrm{Jy}$. As IRS 4 is not covered by our $18 \mu \mathrm{m}$ data we have no measure for the slope of its spectral energy distribution at mid IR wavelengths. However, from its location in the near IR two-colour diagram Frogel et al. (1977) derive a visual extinction of 7.7 , which is slightly larger than the usually adopted $A_{V}$ value of 4.5 towards the OB cluster. Assuming the (old) distance of $8.6 \mathrm{kpc}$ for NGC 3603, the same authors find the dereddened near IR colours and the intrinsic $K$ band magnitude of IRS 4 consistent with those of supergiants of spectral type MIb. Basically, this classification of IRS 4 holds even if we use a revised value of $7 \mathrm{kpc}$ (see Sect. 1) for the distance of NGC 3603.

\subsection{Faint sources in the cluster center}

Apart from the Galactic Center region, the center of the NGC 3603 OB cluster harbours the densest concentration of early-type stars known in the Galaxy. Its core radius of about $0^{\prime \prime} .71(\sim 0.02 \mathrm{pc}$ at the distance of $7 \mathrm{kpc})$ makes NGC 3603 even more extreme than R $136\left(r_{\mathrm{c}} \sim 0^{\prime \prime}\right.$. 82, corresponding to $\sim 0.2 \mathrm{pc}$ at the distance of $50 \mathrm{kpc}$ ), the stellar core of 30 Doradus in the Large Magellanic Cloud (Moffat et al. 1985). Within a radius of 4" Drissen et al. (1995) identify three hydrogen-rich WN6+abs stars (later confirmed as WN6ha stars by Crowther \& Dessart 1998), two O3III(f*) stars, four O3V stars, one $\mathrm{O} 4 \mathrm{~V}$ star, one $\mathrm{O} 5 \mathrm{~V}+\mathrm{OB}($ ?) star, one O6V star, one $\mathrm{O} 6.5 \mathrm{~V}+\mathrm{OB}($ ?) star and one O8V-III star.

Given such a hostile environment, it is a big surprise that close inspection of our $11.9 \mu \mathrm{m}$ data (see Fig. 6) reveals a number of (very) faint mid IR sources in the central region of the NGC 3603 OB cluster. Even better, three of them (namely NS 6A, 6B and 6C) can be unambiguously identified with the above mentioned three Wolf-Rayet (WR) stars WR 43abc (alias MDS 30, MDS 23 and MDS 16; Moffat et al. 1994; alias MTT A1, MTT B and MTT C; Melnick et al. 1989).

\subsubsection{Wolf-Rayet stars}

The $11.9 \mu \mathrm{m}$ fluxes measured for NS 6A and 6B correspond to magnitudes of $N \sim 5$.6. Unfortunately, their individual $K$ band magnitudes are not very well known due to their large brightness which makes them difficult to observe without saturation. Eisenhauer et al. (1998) report $K$-band magnitudes of 7.68 and 7.65 for MDS 30 and MDS 23, respectively. Allowing for saturation or non-linearity of their images, these stars may be even slightly brighter, but certainly not very much brighter as Frogel et al. (1977) report a $K$-band magnitude of 5.73 for the entire cluster center. Thus, the colour of the WR stars seems to be redder than $K-N \sim 1$. Considering a foreground reddening of $A_{V} \sim 4 \mathrm{~m} .5$, which converts to $A_{K} \sim 0.50$ and $A_{N} \sim 0.23$ (Rieke \& Lebofsky 1985), the extinction corrected $K-N$ colour of the WR stars should be of the order 0.7 , at least. Early type main sequence stars, however, have intrinsic $K-N$ colours of more or less 0 . If the reddening within the center of the NGC 3603 cluster behaves normal, it appears likely that we see some small amount of $N$-band excess emission from the WR stars.

Infrared excess emission is a well known feature of WR stars and is generally attributed to four principle mechanisms (see Hackwell et al. 1974): (1) radiation from a cool companion star, (2) line radiation from a circumstellar shell, (3) thermal radiation from an optically thick circumstellar dust shell and (4) free-free radiation from a circumstellar plasma. In our case, the first two arguments can be ruled out quite easily while the latter two may provide plausible explanations.

$\operatorname{MDS} 30(=\mathrm{WR} 43 \mathrm{a})$ is a short-period binary (WN6ha, $P \sim$ $3.77 \mathrm{~d}$; Moffat et al. 1994) and about $40 \%$ of all known Galactic Wolf-Rayet stars of type WN and WC are (probable) binaries, too (see Table 20 of van der Hucht 2001). Taking into account these statistics, an additional late type companion would need a rather large separation to make the whole system stable. However, in the center of the NGC 3603 cluster the existence of companions with long periods is doubtful at all, given the extremely high density of stars there.

As already mentioned in Sect. 3, the throughput of TIMMI2's N11.9 filter drops down below 50\% for wavelengths $\lesssim 11.0 \mu \mathrm{m}$ and $\gtrsim 12.2 \mu \mathrm{m}$. Hence, it appears unlikely that contributions from line emission - in particular, the $\mathrm{H}_{2}$ line at $12.3 \mu \mathrm{m}$ as well as the fine-structure lines of [S IV] at $10.5 \mu \mathrm{m}$ and $[\mathrm{Ne} \mathrm{II}]$ at $12.8 \mu \mathrm{m}$ (see e.g. Smith \& Houck 2001) - from highly excited gas play a significant role for our measured $11.9 \mu \mathrm{m}$ fluxes. This is confirmed by narrow band [Ne II] $12.8 \mu \mathrm{m}$ and [S IV] $10.5 \mu \mathrm{m}$ observations (Lacy et al. 1982; their figures 5 and 6) which give no indication for line emission arising at the cluster center.

Although our imaging data do not allow to distinguish between the two individual components, we assume that the observed mid IR excesses most likely arise from free-free and/or dust emission. According to Hackwell et al. (1974), free-free emission from a circumstellar plasma does considerably contribute to the mid IR fluxes of a large number of WR stars. Similarly, several studies (e.g. Williams et al. 1987, 1994; Usov 1991; Crowther 1997; Marchenko et al. 1999) indicate the possibility of dust production due to colliding stellar winds of WR binaries. Near and mid IR observations at high angular resolution (e.g. most recently for WR 112 and WR 140 by Marchenko et al. 2002 and Monnier et al. 2002, respectively) allowed to measure the sizes of the dust envelopes and revealed arc like and/or clumpy structures within them, which may trace 
the persistent or periodic/episodic formation of dust particles. The involved physical and chemical processes, however, are not yet fully understood (see Cherchneff 1997; Cherchneff et al. 2000).

\subsubsection{Early and late type stars with $N$ excess}

Besides the counterparts to the bright WR stars a number of other mid IR sources are found. A detailed comparison between the mid IR image of the cluster and a VLT + ISAAC $K$-band image of the same area (Nürnberger \& Petr-Gotzens 2002) shows that for many of them $K$-band counterparts can be easily identified. However, these generally do not belong to the brightest stars seen in the cluster, i.e. their $K-N$ colours must be extremely red. In addition, a few mid IR sources do not seem to have a $K$-band counterpart at all, hence their colours must be even more extreme.

The location of the faint mid IR sources close to the center of the NGC 3603 cluster strongly suggest that we see indeed sources within the cluster, rather than background objects like red supergiants or luminous but deeply embedded young stellar objects - in particular, as a wind-blown cavity around the cluster center is mostly void of interstellar gas and dust (Balick et al. 1980; Clayton 1986, 1990). Furthermore, the cluster center itself is presumably by far too evolved to harbour protostars, which are still embedded in their envelope, actively accreting circumstellar material, and which may produce mid IR emission sufficient enough to explain the extreme $K-N$ colours of our sources.

We suggest three different explanations for these mid IR sources. (1) +(2) Analog to the WR stars the $N$-band excesses might be caused by either free-free and/or thermal radiation from highly ionized and/or dusty circumstellar shells. However, such a scenario should only be applicable for the stronger (both in $K$ and $N$ ) sources or for sources right in the center of the cluster, i.e. very close to the energetic WR and $O$ type stars. (3) Alternatively and particularly favourable for the sources with weak or totally missing $K$-band counterpart, the mid IR emission could arise from externally heated circumstellar disks (see Robberto et al. 2002). In the following we discuss this third scenario in some more detail.

In order to get relatively strong mid IR emission as observed for the sources in the cluster, one needs a sufficient amount of circumstellar material heated to temperatures of a few hundred Kelvin. In addition, as mid IR emission gets optically thick fairly quickly, a sufficiently large surface must be present. E.g., in (internally heated) T Tauri star disks the area contributing most to the total $10 \mu \mathrm{m}$ flux is generally a ring, of the order a few AU wide and at a distance from the star of about one to a few AU, i.e. covering a surface area of just a few square AU. Such objects in nearby star forming regions have typical $N$-band fluxes of a few Jy, which translates to a few mJy at the distance of NGC 3603, at least a factor of 10 lower than the fluxes reported in this paper.

The $N$-band flux from a circumstellar disk could be much larger if the entire surface of the disk - typical sizes are several tens to a few hundred $\mathrm{AU}$ in diameter, i.e. the surfaces are of the order 100 square AU or even larger - were heated. We speculate that the $11.9 \mu \mathrm{m}$ sources seen close to the cluster center could represent (remnant) circumstellar disks around cluster members, with large parts of the disk surfaces externally heated by the massive stars in the cluster. As such, these objects would be more similar to the proplyds detected in Orion than the three emission nebulae suggested by Brandner et al. (2000; see also the discussion presented in Sect. 6.8). Therefore, our mid IR observations may provide a hint that at least some (formerly massive) disks may survive even the extreme conditions in a starburst cluster for $10^{6}$ years or more. It might be that the stars at the center of the disks are just massive enough, i.e. provide a sufficiently large gravitational potential, (a) to build up rather massive disks during their own formation process and (b) to keep most of the disk material gravitationally bound despite of the hostile environment. Certainly, this is a very speculative claim. Further observations with higher sensitivity and a larger wavelength coverage are required to verify the existence of such externally heated disks and to constrain the nature of the population of faint mid IR sources found in our survey.

\subsection{The ring nebula around Sher 25}

In Figs. 2 and 6 we have labeled two diffuse emission features NS 17A and NS 17B. Their positions suggest that both are associated with a ring-like nebula, discovered by Brandner et al. (1997a) around the blue supergiant Sher 25. This ring nebula is outlined by the dashed ellipse in Fig. 6.

According to Brandner et al. (1997a), the clumpy ring is tilted by $64^{\circ}$ against the plane of the sky and its semimajor axis (position angle $\sim 165^{\circ}$ ) is $6^{\prime \prime} .9$ long, corresponding to a diameter of about $0.5 \mathrm{pc}$ at the distance of $7 \mathrm{kpc}$. High resolution spectra show that the ring has a systemic velocity of $+19 \mathrm{~km} \mathrm{~s}^{-1}$ (which is in good agreement with the range of radial velocities, from $+9 \mathrm{~km} \mathrm{~s}^{-1}$ to $+20 \mathrm{~km} \mathrm{~s}^{-1}$, measured for NGC 3603's molecular gas by Nürnberger et al. 2002b) and a deprojected expansion velocity of $20 \mathrm{~km} \mathrm{~s}^{-1}$. The high $[\mathrm{N}$ II] / $\mathrm{H} \alpha$ ratio suggests strong $\mathrm{N}$ enrichment.

Recently, Smartt et al. (2002) performed a detailed abundance analysis of Sher 25 . They find that its atmospheric chemistry and wind properties are not peculiar in comparison to those in other galactic B-type supergiants. Furthermore, there is no evidence that the star was previously in a red supergiant phase during which the nebula could have been ejected. However, the post-main sequence evolution of Sher 25 is particularly interesting as its circumstellar nebulae - in addition to the ring there are also bipolar outflow lobes - resemble those observed around SN $1987 \mathrm{~A}$ in many aspects (Brandner et al. 1997a,b).

\subsection{Proplyd-like sources}

Brandner et al. (2000) reported the detection of three small emission line nebulae which are tadpole shaped and suggested to be "proplyds" (protoplanetary disks) like those seen in the Orion Nebula. Accordingly, Brandner et al. (2000) modeled them as disks around young stars, which are photoevaporated 
by the intense ultraviolett (UV) radiation originating from the NGC 3603 OB cluster. However, to account for the observed sizes of the nebulae - which are much larger than the proplyds in Orion - a rather massive $\left(0.86 \mathcal{M}_{\odot}\right.$ for the disk/ envelope system after $5 \times 10^{5} \mathrm{yr}$ ) and large (diameter of $3400 \mathrm{AU}$ ) disk around a central star of $1.14 \mathcal{M}_{\odot}$ is assumed.

Our deep mid IR imaging does not reveal any point-like source which might be associated with one of the three nebulae (see the inserts of Fig. 3). Only "proplyd" 3 to the northwest of the OB cluster shows some faint emission at $11.9 \mu \mathrm{m}$, whose morphology appears to be extended rather than compact (the latter would be expected for emission from a circumstellar accretion disk at such a large distance). Some very faint diffuse emission is also present at the position of "proplyd" 1 but - given its location within more extended emission - it is impossible to tell whether this emission is associated with "proplyd" 1 or not. There is no indication for any mid IR counterpart (either compact or diffuse) of "proplyd" 2 , probably due to the overlapping SF 2 ridge.

Our non-detection of any compact sources associated with NGC 3603's "proplyds" does certainly not rule out the presence of young stellar objects within them. To give an example, shifting an object like T Tau (IRAS $12 \mu \mathrm{m}$ flux $\sim 15 \mathrm{Jy}$, distance about $140 \mathrm{pc}$ ) to a distance of $7 \mathrm{kpc}$ one would receive a flux of only $6 \mathrm{mJy}$, clearly below our detection limit. More massive objects in the same evolutionary stage as T Tau (i.e. in transition from Class I protostar to classical T Tauri stage) or somewhat earlier (corresponding to Class I objects in the low mass case) seem, however, unlikely to exist in the three nebulae as no physically related (!) counterparts at near IR wavelengths are known so far. Only in their youngest evolutionary stage (corresponding to the highly embedded Class 0 phase) protostars of intermediate mass may be present in the "proplyds", but that would demand very high extinction via massive circumstellar envelopes.

These considerations as well as our tentative detection of extended $11.9 \mu \mathrm{m}$ emission from "proplyd" 3 may question the proplyd model for the three nebulae. Rather than being evaporated gas streaming off a large circumstellar disk, it seems more likely that NGC 3603's "proplyds" simply represent small, dense clumps of gas and dust, which are heated and evaporated at their surfaces by the nearby OB stars in a way similar to the much larger and more massive pillars. Thus, both the pillarand "proplyd"-like features may constitute the last remnants of density enhancements, which were present in the original molecular cloud the NGC 3603 OB cluster has formed from (Nürnberger et al. 2002b). This interpretation is supported by the fact that optical (e.g. Fig. 1) and near IR (e.g. Brandl et al. 1999) images reveal even more pillar-like structures within the NGC $3603 \mathrm{H}$ II region, whose sizes (and most likely masses) are settled inbetween those found for the three small "proplyds" and the two large pillars.

As indicated by Brandner et al. (2000) and Nürnberger et al. (2002a), observations with higher sensitivity and/or at higher angular resolution will soon be feasible with the upcoming mid IR instruments on groundbased $8 \mathrm{~m}$ telescopes, like the VLT Imager and Spectrometer for the mid IR (VISIR) and the MID infrared Interferometric instrument (MIDI) at ESO's Very
Large Telescope (Interferometer). In fact, such data would be essential to further constrain the true nature of the "proplyd"like nebulae in NGC 3603.

\subsection{Diffuse emission}

In good agreement with the early results of Frogel et al. (1977) we do not find any significant diffuse $11.9 \mu \mathrm{m}$ or $18 \mu \mathrm{m}$ emission at the center of the OB cluster. Moreover, our mid IR data confirm the picture already developed from optical and near IR data (e.g. Balick et al. 1980; Clayton 1986, 1990; Brandl et al. 1999; Brandner et al. 2000) that stellar winds have freed a cavity of radius $\sim 30^{\prime \prime}-45^{\prime \prime}$ (i.e. $1.0 \mathrm{pc}-1.5 \mathrm{pc}$ at the distance of $7 \mathrm{kpc}$ ) from interstellar material.

\subsubsection{Shocks and ionization fronts}

However, towards the south/south-east and northwest/west/ south-west of the cluster we detect large amounts of diffuse and partly filamentary mid IR emission, which nicely correlates with structures seen at optical (e.g. Fig. 1) and near IR wavelengths (e.g. Nürnberger \& Petr-Gotzens 2002). In particular, one must mention the shock fronts SF 1 to SF 5 (as outlined in Fig. 2) which show flux ratios $F_{11.9 \mu \mathrm{m}} / F_{18 \mu \mathrm{m}}$ of about $0.3-0.5$ (Fig. 4e), corresponding to dust temperatures of a few $100 \mathrm{~K}$. Their overall orientation as well as parts of their substructures are also perfectly traced by narrow band mid IR observations in the fine-structure lines of [S IV] at $10.5 \mu \mathrm{m}$ and [Ne II] at $12.8 \mu \mathrm{m}$ (Lacy et al. 1982; their Fig. 5). Hence, they may consist of swept up material, including both highly excited/ionized gas and heated dust.

\subsubsection{Large pillars}

Additionally, ionisation fronts surrounding the head of the western pillar are clearly detected at both $11.9 \mu \mathrm{m}$ and $18 \mu \mathrm{m}$. They are resolved in several bright arc-like rims, which most likely outline shock-compressed and highly excited gas and dust. Probably, they can be traced towards the south as indicated by the unlabeled dotted lines in Fig. 2 .

Finally and equally noteworthy, the head of the eastern pillar does (in comparison to its western analogue) barely exhibit any rim-like emission from shocked material by itself, but is seen in absorption against the bright mid IR background of shock front SF 2 . This is particularly fascinating, because - in combination with optical and near IR data - it provides us with a vivid 3-dimensional impression of that area and lets the eastern pillar virtually protrude from a "plane" running from SF 2 (in the background of the pillar) to SF 5 (in the foreground of the pillar).

\section{Summary}

Our TIMMI 2 survey of NGC 3603 reveals mid IR emission associated with a large diversity of point sources, including WR stars, evolved early type stars, relatively young late type stars, candidate protostars and maser sources. In addition, we find an impressing complexity (pillars, shocks and ionization fronts) 
in the diffuse component, which documents the huge influence (via stellar winds and ionizing photons) exerted by the massive cluster stars on their surrounding, nearby as well as farther away. This diversity and complexity makes a full understanding of NGC 3603's star formation history and of its still ongoing star formation processes extremely difficult. However, our survey provides an important piece to the entire puzzle.

Without doubt, the proximity of NGC 3603 makes it - although scaled down in size - the best analogue of typical extragalactic starburst regions. It offers the unique opportunity to study in great detail the physical and chemical parameters related to the presence of numerous (relatively young) massive stars. Taking into account these results in the analysis and interpretation of data obtained for more distant and (in most cases certainly) unresolved starburst regions will provide important new insights.

Therefore, our study presented here should be considered as a starting point. Follow-up mid IR observations (both imaging and spectroscopy) with higher angular resolution and higher sensitivity as well as complementary observations at other wavelengths are needed to understand the nature of individual sources in detail and to explain the true origin of their thermal radiation.

Acknowledgements. We would like to thank Kate Brooks, HansUlrich Käufl and Michael Sterzik for their help during the observations. We are grateful to two anonymous referees whose comments helped to improve the presentation of our results. This research has been supported by the Deutsche Forschungsgemeinschaft (DFG) in the framework of its "Physics of Star Formation" program under grants Yo 5/23-1, Zi 242/9-1 and Zi 242/22-2. DEAN also acknowledges financial support by IRAM, Grenoble.

\section{References}

André, P., \& Montmerle, T. 1994, ApJ, 420, 837

Balick, B., Boeshaar, G. O., \& Gull, T. R. 1980, ApJ, 242, 584

Bertero, M., Boccacci, P., \& Robberto, M. 2000, PASP, 112, 1121

Brandl, B., Brandner, W., Eisenhauer, F., et al. 1999, A\&A, 352, L69

Brandner, W., Grebel, E. K., Chu, Y.-H., \& Weis, K. 1997a, ApJ, 475, L45

Brandner, W., Chu, Y.-H., Eisenhauer, F., Grebel, E. K., \& Points, S. D. 1997b, ApJ, 489, L153

Brandner, W., Grebel, E. K., Chu, Y.-H., et al. 2000, AJ, 119, 292

Braz, M. A., \& Scalise, E. 1982, A\&A, 107, 272

Braz, M. A., \& Epchtein, N. 1983, A\&AS, 54, 167

Caswell, J. L. 1998, MNRAS, 297, 215

Caswell, J. L., \& Haynes, R. F. 1987, Aust. J. Phys., 40, 215

Caswell, J. L., Batchelor, R. A., Forster, J. R., \& Wellington, K. J. 1989, Aust. J. Phys., 42, 331

Caswell, J. L., Vaile, R. A., Ellingsen, S. P., Whiteoak, J. B., \& Norris, R. P. 1995, MNRAS, 272, 96

Cherchneff, I. 1997, Ap\&SS, 251, 333

Cherchneff, I., Le Teuff, Y. H., Williams, P. M., \& Tielens, A. G. G. M. 2000, A\&A, 357, 572

Clayton, C. A. 1986, MNRAS, 219, 895

Clayton, C. A. 1990, MNRAS, 246, 712

Cohen, M. 1998, AJ, 115, 2092

Crowther, P. A. 1997, MNRAS, 290, L59

Crowther, P. A., \& Dessart, L. 1998, MNRAS, 296, 622
De Pree, C. G., Nysewander, M. C., \& Goss, W. M. 1999, AJ, 117, 2902

Drissen, L., Moffat, A. F. J., Walborn, N. R., \& Shara, M. M. 1995, AJ, 110, 2235

Ducati, J. R., Bevilacqua, C. M., Rembold, S. B., \& Ribeiro, D. 2001, ApJ, 558, 309

Eisenhauer, F., Quirrenbach, A., Zinnecker, H., \& Genzel, R. 1998, ApJ, 498, 278

Frogel, J. A., Persson, S. E., \& Aaronson, M. 1977, ApJ, 213, 723

Georgelin, Y. P., \& Georgelin, Y. M. 1970, A\&A, 7, 133

Georgelin, Y. M., Russeil, D., Amram, P., et al. 2000, A\&A, 357, 308

Goss, W. M., \& Radhakrishnan, V. 1969, ApL, 4, 199

Goss, W. M., Radhakrishnan, V., Brooks, J. W., \& Murray, J. D. 1972, ApJS, 24, 123

Hackwell, J. A., Gehrz, R. D., \& Smith, J. R. 1974, ApJ, 192, 383

Hofmann, K.-H., \& Weigelt, G. 1986, A\&A, 167, L15

Hofmann, K.-H., Seggewiss, W., \& Weigelt, G. 1995, A\&A, 300, 403

Kennicutt, R. C. 1984, ApJ, 287, 116

Koornneef, J. 1983a, A\&AS, 51, 489

Koornneef, J. 1983b, A\&A, 128, 84

Lacy, J. H., Beck, S. C., \& Geballe, T. R. 1982, ApJ, 255, 523

Lada, C. J. 1987, in Star Forming Regions, ed. M. Peimbert, \& J. Jugaku, IAU Symp., 115, 1

Marchenko, S. V., Moffat, A. F. J., \& Grosdidier, Y. 1999, ApJ, 522, 433

Marchenko, S. V., Moffat, A. F. J., Vacca, W. D., Côté, S., \& Doyon, R. 2002, ApJ, 565, L59

Melnick, J., Tapia, M., \& Terlevich, R. 1989, A\&A, 213, 89

Moffat, A. F. J. 1974, A\&A, 35, 315

Moffat, A. F. J. 1983, A\&A, 124, 273

Moffat, A. F. J., Seggewiss, W., \& Shara, M. M. 1985, ApJ, 295, 109

Moffat, A. F. J., Drissen, L., \& Shara, M. M. 1994, ApJ, 436, 183

Monnier, J. D., Tuthill, P. G., \& Danchi, W. C. 2002, ApJ, 567, L137

Nürnberger, D. E. A. 2002, in Hot Star Workshop III: The Earliest Phases of Massive Star Birth, ed. P. A. Crowther, ASP Conf. Ser., 267, 127

Nürnberger, D. E. A. 2003, A\&A, submitted

Nürnberger, D. E. A., \& Petr-Gotzens, M. G. 2002, A\&A, 382, 537

Nürnberger, D., Bronfman, L., Petr-Gotzens, M., \& Stanke, Th. 2002a, in The Origin of Stars and Planets: The VLT View, ed. J. Alves, \& M. McCaughrean, ESO Astrophys. Symp. Ser., 297

Nürnberger, D. E. A., Bronfman, L., Yorke, H. W., \& Zinnecker, H. 2002b, A\&A, 394, 253

Pandey, A. K., Ogura, K., \& Sekiguchi, K. 2000, PASJ, 52, 847

Reimann, H.-G., Linz, H., Wagner, R., et al. 2000, SPIE, 4008, 1132

Rieke, G. H., \& Lebofsky, M. J. 1985, ApJ, 288, 618

Robberto, M., Beckwith, S. V. W., \& Panagia, N. 2002, ApJ, 578, 897

Roth, M., Tapia, M., Ruiz, M. T., Persi, P., \& Ferrari-Toniolo, M. 1987, in Star Forming Regions, ed. M. Peimbert, \& J. Jugaku, IAU Symp., 115, 182

Sagar, R., Munari, U., \& de Boer, K. S. 2001, MNRAS, 327, 23

Smartt, S. J., Lennon, D. J., Kudritzki, R. P., et al. 2002, A\&A, 391, 979

Smith, J. D. J., \& Houck, J. R. 2001, AJ, 121, 2115

Tapia, M. 1981, MNRAS, 197, 949

Tapia, M., Bohigas, J., Pérez, B., Roth, M., \& Ruiz, M. T. 2001, RMxAA, 37, 39

Usov, V. V. 1991, MNRAS, 252, 49

Van den Bergh, S. 1978, A\&A, 63, 275

van den Boos, W. H. 1928, Bull. Astron. Inst. Netherlands, 4, 261

van der Hucht, K. A. 2001, NewAR, 45, 135

Williams, P. M., van der Hucht, K. A., \& Thé, P. S. 1987, A\&A, 182, 91

Williams, P. M., van der Hucht, K. A., Kidger, M. R., Geballe, T. R., \& Bouchet, P. 1994, MNRAS, 266, 247 